\title{
Pacific
}

Journal of

Mathematics

\section{SEMIDIRECT PRODUCTS OF REPRESENTATIONS UP TO} HOMOTOPY

\author{
Yunhe SHENG AND CHENCHANG ZHU
}




\title{
SEMIDIRECT PRODUCTS OF REPRESENTATIONS UP TO HOMOTOPY
}

\author{
Yunhe SHENG AND CHENCHANG ZHU
}

\begin{abstract}
We study the semidirect product of a Lie algebra with a representation up to homotopy and provide various examples coming from Courant algebroids, Lie 2-algebras of string type, and omni-Lie algebroids. In the end, we study the semidirect product of a Lie group with a representation up to homotopy and use it to give an integration of a certain Lie 2-algebra of string type.
\end{abstract}

\section{Introduction}

This paper is the first part of our project to integrate representations up to homotopy of Lie algebras (algebroids). Our original motivation is to integrate the standard Courant algebroid $T M \oplus T^{*} M$, since it is this Courant algebroid that is much used in Hitchin and Gualtieri's program of generalized complex geometry. Courant algebroids are Lie 2-algebroids in the sense of Roytenberg [2002] and Ševera [2005]. The general procedure to integrate Lie $n$-algebras (algebroids) is already described in [Getzler 2009; Henriques 2008; Ševera 2005]. We want to pursue some explicit formulas for the special case of the standard Courant algebroid. It turns out that the sections of the Courant algebroid $T M \oplus T^{*} M$ form a semidirect product of a Lie algebra with a representation up to homotopy. Abad and Crainic [2009] recently studied the representations up to homotopy of Lie algebras, Lie groups, and even Lie algebroids, Lie groupoids, in general. Just as one can form the semidirect product of a Lie algebra with a representation, one can form the semidirect product with representations up to homotopy too. In our case, the semidirect product coming from the standard Courant algebra is a Lie 2-algebra. But using the fact that it is also a semidirect product, the integration becomes easier. The integration result is related to the semidirect product of Lie groups with its representation up to homotopy, as will be discussed in Section 3. However it turns out that Abad

MSC2000: primary 17B65; secondary 18B40, 58H05.

Keywords: representation up to homotopy, $L_{\infty}$-algebra, integration, Lie 2-algebras, Courant algebroids.

Supported by the German Research Foundation (Deutsche Forschungsgemeinschaft (DFG)) through the Institutional Strategy of the University of Göttingen, NSF of China (10871007) and China Postdoctoral Science Foundation (20090451267). 
and Crainic's concept of representations up to homotopy of Lie groups will not be general enough to cover all the integration results. This we will continue in a forthcoming paper [Sheng and Zhu 2010].

In this paper we focus on exhibiting more examples of representations up to homotopy and their semidirect products in order to demonstrate the importance of our integration procedure. The examples are all variations of Courant algebroids. One is Chen and Liu's omni-Lie algebroids, which generalize Weinstein's omniLie algebras. Hence we expect in [Sheng and Zhu 2010] to give an integration to Weinstein's omni-Lie algebras via Lie 2-algebras.

Another example comes from a more general string Lie 2-algebra, which we call the Lie 2-algebra of string type. It is essentially a Courant algebroid over a point (see Example II), namely a Lie algebra with an adjoint-invariant inner product. This sort of Lie algebra is usually called a quadratic Lie algebra. This concept also appears in the context of Manin triples and double Lie algebras. The example $\mathbb{R} \rightarrow \mathfrak{g} \oplus \mathfrak{g}^{*}$ that we consider in this paper is an analogue of the standard Courant algebroid, and is basically a special case taken from [Lu and Weinstein 1990]. ${ }^{1}$ We give an integration of the Lie 2-algebra of string type $\mathbb{R} \rightarrow \mathfrak{g} \oplus \mathfrak{g}^{*}$ at the end.

Usually people require the base Lie algebra of a string Lie 2-algebra to be semisimple and of compact type (see Remark 2.11). For such string Lie 2-algebras, Baez and Lauda [2004] have proved a no-go theorem: such string Lie 2-algebras cannot be integrated to finite-dimensional semistrict Lie 2-groups. Here a semistrict Lie 2-group is a group object in DiffCat, where DiffCat is the 2-category consisting of categories, functors, and natural transformations in the category of differential manifolds, or equivalently DiffCat is a 2-category with Lie groupoids as objects, strict morphisms of Lie groupoids as morphisms, and 2-morphisms of Lie groupoids as 2-morphisms. Our semistrict Lie 2-group is actually called a Lie 2-group by Baez and Lauda. However, we call it a semistrict Lie 2-group because compared to the Lie 2-group in the sense of Henriques [2008], or equivalently (the equivalency was proved in [Zhu 2009]) the stacky group in the sense of Blohmann [2008], it is stricter. Basically, their Lie 2-group is a group object in the 2-category with objects as Lie groupoids, morphisms as Hilsum-Skandalis bimodules (or generalized morphisms), 2-morphisms as 2-morphisms of Lie groupoids. Schommer-Pries [2010] realizes the string 2-group as such a Lie 2-group with a finite-dimensional model; the integration of a string Lie 2-algebra to such a model is work in progress [Schommer-Pries et al. $\geq 2011$ ].

It is not needed in the definition of the string Lie 2-algebra for the base Lie algebra to be semisimple of compact type. One only needs a quadratic Lie algebra. As soon as we relax this condition, we find out that one can integrate $\mathbb{R} \rightarrow \mathfrak{g} \oplus \mathfrak{g}^{*}$

${ }^{1}$ private conversation with Jiang-Hua Lu 
to a finite dimensional semistrict Lie 2-group in the sense of Baez and Lauda. The integrating object is actually a special Lie 2-group (very close to a strict Lie 2-group) in the sense of Baez and Lauda.

Of course, as we relax the condition, we are in danger that the class corresponding to this Lie 2-algebra in $H^{3}\left(\mathfrak{g} \oplus \mathfrak{g}^{*}, \mathbb{R}\right)$ might be trivial, and consequently our Lie 2-algebra might be trivially strictified. Then what we have done would not have been a big surprise because a strict Lie 2-algebra corresponds to a crossed module of Lie algebras, and it easily integrates to a strict Lie 2-group by integrating the crossed module. However, we verify that when $\mathfrak{g}$ itself (not $\mathfrak{g} \oplus \mathfrak{g}^{*}$ ) is semisimple, this class is not trivial.

\section{Representations up to homotopy of Lie algebras}

Here, we first consider the 2-term representation up to homotopy of Lie algebras. We give explicit formulas of the corresponding 2-term $L_{\infty}$-algebra, which is their semidirect product. Then we give several interesting examples including Courant algebroids and omni-Lie algebroids.

\section{2a. Representations up to homotopy of Lie algebras and their semidirect prod-} ucts. $L_{\infty}$-algebras, sometimes called strongly homotopy Lie algebras, were introduced by Drinfeld and Stasheff [Stasheff 1992] as a model for "Lie algebras that satisfy Jacobi identity up to all higher homotopies". The following convention of $L_{\infty}$-algebras has the same grading as in [Henriques 2008] and [Roytenberg and Weinstein 1998].

Definition 2.1. An $L_{\infty}$-algebra is a graded vector space $L=L_{0} \oplus L_{1} \oplus \cdots$ equipped with a system $\left\{l_{k} \mid 1 \leq k<\infty\right\}$ of linear maps $l_{k}: \bigwedge^{k} L \rightarrow L$ with degree $\operatorname{deg}\left(l_{k}\right)=$ $k-2$, where the exterior powers are interpreted in the graded sense and the sum

$$
\sum_{i+j=n+1}(-1)^{i(j-1)} \sum_{\sigma} \operatorname{sgn}(\sigma) \operatorname{Ksgn}(\sigma) l_{j}\left(l_{i}\left(x_{\sigma(1)}, \ldots, x_{\sigma(i)}\right), x_{\sigma(i+1)}, \ldots, x_{\sigma(n)}\right)
$$

vanishes for all $n \geq 0$, where Ksgn is the Koszul sign and the sum is taken over all $(i, n-i)$-unshuffles with $i \geq 1$.

Letting $n=1$, we have

$$
l_{1}^{2}=0, \quad l_{1}: L_{i+1} \rightarrow L_{i},
$$

which means that $L$ is a complex; we usually write $\mathrm{d}=l_{1}$. Letting $n=2$, we have

$$
\mathrm{d} l_{2}(x, y)=l_{2}(\mathrm{~d} x, y)+(-1)^{p} l_{2}(x, \mathrm{~d} y) \quad \text { for all } x \in L_{p}, y \in L_{q},
$$

which means that $\mathrm{d}$ is a derivation with respect to $l_{2}$. We usually view $l_{2}$ as a bracket $[\cdot, \cdot]$. However, it is not a Lie bracket: the obstruction of the Jacobi 
identity is controlled by $l_{3}$ :

$$
\begin{aligned}
& l_{2}\left(l_{2}(x, y), z\right)+(-1)^{(p+q) r} l_{2}\left(l_{2}(y, z), x\right)+(-1)^{q r+1} l_{2}\left(l_{2}(x, z), y\right) \\
& =-\mathrm{d} l_{3}(x, y, z)-l_{3}(\mathrm{~d} x, y, z)+(-1)^{p q} l_{3}(\mathrm{~d} y, x, z)-(-1)^{(p+q) r} l_{3}(\mathrm{~d} z, x, y),
\end{aligned}
$$

where $x \in L_{p}, y \in L_{q}, \quad z \in L_{q}$ and $l_{3}$ also satisfies higher coherence laws.

In particular, if the $k$-th brackets are zero for all $k>2$, we recover the usual notion of differential graded Lie algebras (DGLA). If $L$ is concentrated in degrees less than $n$, then $L$ is called an $n$-term $L_{\infty}$-algebra.

In this paper, we mainly consider 2-term $L_{\infty}$-algebras, which are equivalent to the Lie 2-algebras given by John Baez and Alissa Crans [2004]. In this special case, $l_{4}$ is always zero. Thus by restricting the coherence law satisfied by $l_{3}$ on degree 0 , we obtain

$$
l_{3}\left(l_{2}(x, y), z, w\right)+\text { c.p. }-\left(l_{2}\left(l_{3}(x, y, z), w\right)+\text { c.p. }\right)=0
$$

for all $x, y, z, w \in L_{0}$,

where c.p. stands for cyclic permutation. Lie 2-algebras are categorified versions of Lie algebras. In a Lie 2-algebra, the Jacobi identity is replaced by an isomorphism called the Jacobiator. The Jacobiator satisfies a certain law of its own. Given a 2-term $L_{\infty}$-algebra $L_{1} \stackrel{\mathrm{d}}{\rightarrow} L_{0}$, the underlying 2-vector space of the corresponding Lie 2-algebra is made up by $L_{0}$ as the vector space of objects and $L_{0} \oplus L_{1}$ as the vector space of morphisms. See [Baez and Crans 2004, Theorem 4.3.6] for details.

Recall from [Baez and Crans 2004] that a Lie 2-algebra is skeletal if isomorphic objects are equal. Viewing a Lie 2 -algebra as a 2 -term $L_{\infty}$-algebra, explicitly we have this:

Definition 2.2. A 2-term $L_{\infty}$-algebra $L_{1} \stackrel{\mathrm{d}}{\longrightarrow} L_{0}$ is called skeletal if $\mathrm{d}=0$.

Theorem 2.3 [Baez and Crans 2004]. There is a bijection between 2-term skeletal $L_{\infty}$-algebra $L_{1} \stackrel{\mathrm{d}}{\rightarrow} L_{0}$ and quadruples $\left(\mathfrak{k}_{1}, \mathfrak{k}_{2}, \phi, \theta\right)$, where $\mathfrak{k}_{1}$ is a Lie algebra, $\mathfrak{k}_{2}$ is a vector space, $\phi$ is a representation of $\mathfrak{k}_{1}$ on $\mathfrak{k}_{2}$, and $\theta$ is a 3 -cocycle on $\mathfrak{k}_{1}$ with values in $\mathfrak{k}_{2}$.

Given a 2 -term skeletal $L_{\infty}$-algebra $L_{1} \stackrel{\mathrm{d}}{\rightarrow} L_{0}$, recall that $\mathfrak{k}_{1}$ is $L_{0}, \mathfrak{k}_{2}$ is $L_{1}$, the representation $\phi$ comes from $l_{2}$, and the 3 -cocycle $\theta$ is obtained from $l_{3}$.

See [Abad and Crainic 2009; Abad 2008] for the general theory of representation up to homotopy of Lie algebroids. In this paper we only consider the 2-term representations up to homotopy of Lie algebras.

Definition 2.4 [Abad and Crainic 2009]. A 2-term representation up to homotopy of a Lie algebra $\mathfrak{g}$ consists of the following:

(i) A 2-term complex of vector spaces $V_{1} \stackrel{\text { d }}{\rightarrow} V_{0}$. 
(ii) Two linear maps $\mu_{i}: \mathfrak{g} \rightarrow \operatorname{End}\left(V_{i}\right)$ that are compatible with d. That is, for any $X \in \mathfrak{g}$ and $\xi \in V_{1}$, we have

$$
\mathrm{d} \circ \mu_{1}(X)(\xi)=\mu_{0}(X) \circ \mathrm{d}(\xi) .
$$

(iii) A linear map $v: \bigwedge^{2} \mathfrak{g} \rightarrow \operatorname{Hom}\left(V_{0}, V_{1}\right)$ such that

$$
\begin{aligned}
& \mu_{0}\left[X_{1}, X_{2}\right]-\left[\mu_{0}\left(X_{1}\right), \mu_{0}\left(X_{2}\right)\right]=\mathrm{d} \circ v\left(X_{1}, X_{2}\right), \\
& \mu_{1}\left[X_{1}, X_{2}\right]-\left[\mu_{1}\left(X_{1}\right), \mu_{1}\left(X_{2}\right)\right]=v\left(X_{1}, X_{2}\right) \circ \mathrm{d},
\end{aligned}
$$

and

$$
\left[\mu_{0}\left(X_{1}\right)+\mu_{1}\left(X_{1}\right), v\left(X_{2}, X_{3}\right)\right]+\text { c.p. }=v\left(\left[X_{1}, X_{2}\right], X_{3}\right)+\text { c.p. }
$$

We usually write $\mu=\mu_{0}+\mu_{1}$ and denote a 2-term representation up to homotopy of a Lie algebra $\mathfrak{g}$ by $\left(V_{1} \stackrel{\mathrm{d}}{\rightarrow} V_{0}, \mu, v\right)$.

In [2009, Example 3.25], Abad and Crainic proved that one can associate to any representation up to homotopy $V_{\bullet}$ of a Lie algebra $\mathfrak{g}$ a new $L_{\infty}$-algebra $\mathfrak{g} \ltimes V_{\bullet}$, which is their semidirect product. Here we make this construction explicit in the 2-term case.

Let $\left(V_{1} \stackrel{\mathrm{d}}{\rightarrow} V_{0}, \mu, v\right)$ be a 2 -term representation up to homotopy of $\mathfrak{g}$. Then we can form a new 2-term complex

$$
\left(\mathfrak{g} \ltimes V_{\bullet}, \mathrm{d}\right): V_{1} \stackrel{\mathrm{d}}{\longrightarrow}\left(\mathfrak{g} \oplus V_{0}\right) .
$$

Define $l_{2}: \bigwedge^{2}\left(\mathfrak{g} \ltimes V_{\bullet}\right) \rightarrow \mathfrak{g} \ltimes V_{\bullet}$ by setting

$$
\begin{aligned}
l_{2}(X+\xi, Y+\eta) & =[X, Y]+\mu_{0}(X)(\eta)-\mu_{0}(Y)(\xi), \\
l_{2}(X+\xi, f) & =\mu_{1}(X)(f), \\
l_{2}(f, g) & =0
\end{aligned}
$$

for any $X+\xi, Y+\eta \in \mathfrak{g} \oplus V_{0}$ and $f, g \in V_{1}$. Note that $l_{2}$ is not a Lie bracket, but instead

$$
l_{2}\left(l_{2}(X+\xi, Y+\eta), Z+\gamma\right)+\text { c.p. }=\mathrm{d}(\nu(X, Y)(\gamma))+\text { c.p. }
$$

Define $l_{3}: \bigwedge^{3}\left(\mathfrak{g} \ltimes V_{\bullet}\right) \rightarrow \mathfrak{g} \ltimes V_{\bullet}$ by setting

$$
l_{3}(X+\xi, Y+\eta, Z+\gamma)=-v(X, Y)(\gamma)+\text { c.p. }
$$

Then:

Proposition 2.5. With the notations above, if $\left(V_{1} \stackrel{\mathrm{d}}{\longrightarrow} V_{0}, \mu, v\right)$ is a 2-term representation up to homotopy of a Lie algebra $\mathfrak{g}$, then $\left(V_{1} \stackrel{\mathrm{d}}{\longrightarrow}\left(\mathfrak{g} \oplus V_{0}\right), l_{2}, l_{3}\right)$ is a 2-term $L_{\infty}$-algebra. 
Example I (Courant algebroids $T M \oplus T^{*} M$ ). Courant algebroids, introduced in [Liu et al. 1997] to study the double of Lie bialgebroids, each consist of a vector bundle $E \rightarrow M$ equipped with a nondegenerate symmetric bilinear form $\langle\cdot, \cdot\rangle$ on the bundle, an antisymmetric bracket $\llbracket \cdot, \cdot \rrbracket$ on the section space $\Gamma(E)$ and a bundle map $\rho: E \rightarrow T M$ such that a set of axioms are satisfied. One can be viewed as a Lie 2-algebroid with a "degree-2 symplectic form" [Roytenberg 2002]. The first example is the standard Courant algebroid $\left(\mathcal{T}=T M \oplus T^{*} M,\langle\cdot, \cdot\rangle, \llbracket \cdot, \cdot \mathbb{1}, \rho\right)$ associated to a manifold $M$, where $\rho: \mathscr{T} \rightarrow T M$ is the projection, the canonical pairing $\langle\cdot, \cdot\rangle$ is given by

$$
\langle X+\xi, Y+\eta\rangle=\frac{1}{2}(\xi(Y)+\eta(X)) \text { for all } X, Y \in \mathfrak{X}(M), \xi, \eta \in \Omega^{1}(M),
$$

and the antisymmetric bracket $\llbracket \cdot, \cdot \rrbracket$ is given by

$$
\begin{aligned}
\llbracket X+\xi, Y+\eta \rrbracket \triangleq[X, Y]+L_{X} \eta-L_{Y} \xi+\frac{1}{2} d(\xi(Y)-\eta(X)) \\
\\
\text { for all } X+\xi, Y+\eta \in \Gamma(\mathscr{T}) .
\end{aligned}
$$

This is not a Lie bracket, but we have

$$
\llbracket \llbracket e_{1}, e_{2} \rrbracket, e_{3} \rrbracket+\text { c.p. }=d T\left(e_{1}, e_{2}, e_{3}\right) \quad \text { for all } e_{1}, e_{2}, e_{3} \in \Gamma(\mathscr{T}),
$$

where $T\left(e_{1}, e_{2}, e_{3}\right)$ is given by

$$
T\left(e_{1}, e_{2}, e_{3}\right)=\frac{1}{3}\left(\left\langle\llbracket e_{1}, e_{2} \rrbracket, e_{3}\right\rangle+\text { c.p. }\right) .
$$

Now we realize the section space of $\mathscr{T}$ as the semidirect product of the Lie algebra $\mathfrak{X}(M)$ of vector fields with the natural 2-term deRham complex

$$
C^{\infty}(M) \stackrel{d}{\longrightarrow} \Omega^{1}(M) .
$$

For this we need to define a representation up to homotopy of $\mathfrak{X}(M)$ on this complex. For any $X \in \mathfrak{X}(M)$, define linear actions $\mu_{0}$ and $\mu_{1}$ by

$$
\begin{array}{ll}
\mu_{0}(X)(\xi) \triangleq \llbracket X, \xi \rrbracket=L_{X} \xi-\frac{1}{2} d(\xi(X)) & \text { for all } \xi \in \Omega^{1}(M), \\
\mu_{1}(X)(f) \triangleq\langle X, d f\rangle=\frac{1}{2} X(f) & \text { for all } f \in C^{\infty}(M) .
\end{array}
$$

Define $v: \bigwedge^{2} \mathfrak{X}(M) \rightarrow \operatorname{Hom}\left(\Omega^{1}(M), C^{\infty}(M)\right)$ by

$$
v(X, Y)(\xi)=T(X, Y, \xi) \quad \text { for all } X, Y \in \mathfrak{X}(M), \xi \in \Omega^{1}(M) .
$$

Proposition 2.6. With the above notations, $\left(C^{\infty}(M) \stackrel{d}{\longrightarrow} \Omega^{1}(M), \mu=\mu_{0}+\mu_{1}, v\right)$ is a representation up to homotopy of the Lie algebra $\mathfrak{X}(M)$.

Proof. For any $f \in C^{\infty}(M)$, we have

$$
\mu_{0}(X)(d f)=L_{X} d f-\frac{1}{2} d X(f)=\frac{1}{2} d X(f),
$$


which implies $\mu_{0} \circ d=d \circ \mu_{1}$, that is, the $\mu_{i}$ are compatible with the differential $d$. By straightforward computations, we have

$$
\begin{aligned}
\mu_{0}[X, Y](\xi)-\left[\mu_{0}(X), \mu_{0}(Y)\right](\xi) & =\llbracket \llbracket X, Y \rrbracket, \xi \rrbracket+\text { c.p. } \\
& =d T(X, Y, \xi)=d(v(X, Y)(\xi)), \\
\mu_{1}[X, Y](f)-\left[\mu_{1}(X), \mu_{1}(Y)\right](f) & =\frac{1}{2}[X, Y](f)-\frac{1}{4}(X(Y(f))-Y(X(f))) \\
& =\frac{1}{4}[X, Y](f), \\
v(X, Y)(d f)=T(X, Y, d f) & =\frac{1}{3}\left(\frac{1}{2}[X, Y](f)+\frac{1}{4}(X(Y(f))-Y(X(f)))\right. \\
& =\frac{1}{4}[X, Y](f),
\end{aligned}
$$

which implies (4) and (5). At last we need to prove (6), which is obviously equivalent to

$$
\begin{aligned}
\mu_{1}(X) T(Y, Z, \xi)-T\left(Y, Z, \mu_{0}(X)(\xi)\right) & + \text { c.p. }(X, Y, Z) \\
& =T([X, Y], Z, \xi)+\text { c.p. }(X, Y, Z) .
\end{aligned}
$$

Observe that since $\mu_{0}(X)(\xi)=\llbracket X, \xi \rrbracket$, we have

$$
\begin{array}{r}
T\left(Y, Z, \mu_{0}(X)(\xi)\right)+\text { c.p. }(X, Y, Z)+T([X, Y], Z, \xi)+\text { c.p. }(X, Y, Z) \\
=T([X, Y], Z, \xi)+\text { c.p. }(X, Y, Z, \xi) .
\end{array}
$$

Furthermore, since $\mu_{1}(X)(f)=\langle X, d f\rangle$ for any $f \in C^{\infty}(M)$ and the cotangent bundle $T^{*} M$ is isotropic under the pairing (9), we have

$$
\mu_{1}(X) T(Y, Z, \xi)+\text { c.p. }(X, Y, Z)=\langle X, d T(Y, Z, \xi)\rangle+\text { c.p. }(X, Y, Z, \xi) .
$$

Thus, (17) is equivalent to

$$
\langle X, d T(Y, Z, \xi)\rangle+\text { c.p. }(X, Y, Z, \xi)=T([X, Y], Z, \xi)+\text { c.p. }(X, Y, Z, \xi),
$$

which holds by [Roytenberg and Weinstein 1998, Lemma 4.5].

By Proposition 2.5, we have this:

Corollary 2.7. $\left(C^{\infty}(M) \stackrel{\mathrm{d}=0 \oplus d}{\longrightarrow}\left(\mathfrak{X}(M) \oplus \Omega^{1}(M)\right), l_{2}, l_{3}\right)$ is a 2-term $L_{\infty}$-algebra, where $l_{2}$ and $l_{3}$ are given by (7) and (8), in which $\mu_{0}, \mu_{1}$ and $v$ are given by (14), (15) and (16), respectively.

Remark 2.8. Roytenberg and Weinstein [1998] have proved that the sections of a Courant algebroid $(\mathscr{C},\langle\cdot, \cdot\rangle, \llbracket \cdot, \cdot \rrbracket, \rho)$ form an $L_{\infty}$-algebra. In the case when $\mathscr{C}=\mathscr{T}$ the standard Courant algebroid, the 2-term $L_{\infty}$-algebra is given by

$$
C^{\infty}(M) \stackrel{\mathrm{d}=0 \oplus d}{\longrightarrow} \mathfrak{X}(M) \oplus \Omega^{1}(M)=\Gamma(\mathscr{T}),
$$


with brackets given by

$$
l_{2}\left(e_{1}, e_{2}\right)=\llbracket e_{1}, e_{2} \rrbracket, \quad l_{2}\left(e_{1}, f\right)=\left\langle e_{1}, \mathrm{~d} f\right\rangle, \quad l_{3}\left(e_{1}, e_{2}, e_{3}\right)=-T\left(e_{1}, e_{2}, e_{3}\right),
$$

and $l_{i \geq 4}=0$ for any $e_{1}, e_{2}, e_{3} \in \Gamma(\mathscr{T})$ and $f \in C^{\infty}(M)$. Here $T$ is defined by (12). It is easy to verify that this is the same as our 2-term $L_{\infty}$-algebra in Corollary 2.7.

We can also modify our complex $(13)$ to $\Omega^{1}(M) \stackrel{\mathrm{Id}}{\longrightarrow} \Omega^{1}(M)$. Following the same procedure, we get another representation up to homotopy of the Lie algebra $\mathfrak{X}(M)$ and therefore obtain another 2-term $L_{\infty}$-algebra that is also totally determined by the Courant algebroid $(\mathscr{T},\langle\cdot, \cdot\rangle, \mathbb{[} \cdot, \cdot \rrbracket, \rho)$. More precisely, $\mu_{0}=\mu_{1}$ is given by

$$
\mu_{0}(X)(\xi) \triangleq \llbracket X, \xi \rrbracket,
$$

and $v: \mathfrak{X}(M) \rightarrow \Omega^{2}\left(\mathfrak{X}^{2}(M), \operatorname{End}\left(\Omega^{1}(M), \Omega^{1}(M)\right)\right)$ is given by

$$
v(X, Y)(\xi) \triangleq d T(X, Y, \xi)
$$

for any $X, Y \in \mathfrak{X}(M)$ and $\xi \in \Omega^{1}(M)$.

Proposition 2.9. With the above notations, $\left(\Omega^{1}(M) \stackrel{\mathrm{Id}}{\longrightarrow} \Omega^{1}(M), \mu=\mu_{0}=\mu_{1}, v\right)$ is a representation up to homotopy of the Lie algebra $\mathfrak{X}(M)$.

Example II (Courant algebroids over a point and Lie 2-algebras of string type). A Courant algebroid over a point is literally a quadratic Lie algebra, namely a Lie algebra $\mathfrak{k}$ together with nondegenerate inner product $\langle\cdot, \cdot\rangle$ that is invariant under the adjoint action. People often think of a Courant algebroid over a point as a string Lie 2 -algebra. ${ }^{2}$ Here we justify this thinking.

Definition 2.10. The Lie 2-algebra of string type associated to a quadratic Lie algebra $(\mathfrak{k},\langle\cdot, \cdot\rangle)$ is a 2 -term $L_{\infty}$-algebra $\mathbb{R} \stackrel{0}{\longrightarrow} \mathfrak{k}$, whose degree- 0 part is $\mathfrak{k}$ and whose degree- 1 part is $\mathbb{R}$, with $l_{2}, l_{3}$ given by

$$
\begin{aligned}
l_{2}\left(\left(e_{1}, c_{1}\right),\left(e_{2}, c_{2}\right)\right) & =\left(\left[e_{1}, e_{2}\right], 0\right), \\
l_{3}\left(\left(e_{1}, c_{1}\right),\left(e_{2}, c_{2}\right),\left(e_{3}, c_{3}\right)\right) & =\left(0,\left\langle\left[e_{1}, e_{2}\right], e_{3}\right\rangle\right),
\end{aligned}
$$

where $e_{1}, e_{2}, e_{3} \in \mathfrak{k}$ and $c_{1}, c_{2}, c_{3} \in \mathbb{R}$.

The representation $\phi$ of $\mathfrak{k}$ on $\mathbb{R}$ and the 3 -cocycle $\theta: \bigwedge^{3} \mathfrak{k} \rightarrow \mathbb{R}$ in the corresponding quadruple in Theorem 2.3 is given by

$$
\begin{aligned}
\rho(e)(c) & =l_{2}(e, c), \\
\theta\left(e_{1}, e_{2}, e_{3}\right) & =\left\langle\left[e_{1}, e_{2}\right], e_{3}\right\rangle .
\end{aligned}
$$

\footnotetext{
${ }^{2}$ Private conversation with John Baez and Urs Schreiber.
} 
Remark 2.11. In the definition of string Lie 2-algebras [Baez and Rogers 2010; Henriques 2008], the base Lie algebra $\mathfrak{k}$ is usually required to be semisimple and of compact type, such that the Jacobiator gives rise to the generator of $H^{3}(\mathfrak{k}, \mathbb{Z})=\mathbb{Z}$. This is because Witten's original motivation was to obtain a 3-connected cover of $\operatorname{Spin}(n)$, and $\mathfrak{s o}(n)$ is simple and of compact type. However, to write down the structure of the string Lie 2-algebra, we only need a quadratic Lie algebra. This is how we obtain the definition above on Lie 2-algebras of string type. Then $H^{3}(\mathfrak{k}, \mathbb{Z})$ is not necessarily $\mathbb{Z}$ for a general quadratic Lie algebra $\mathfrak{k}$. For example, for the abelian Lie algebra $\mathbb{R}$, any inner product is adjoint-invariant, and $H^{3}(\mathbb{R}, \mathbb{Z})=0$. We thus face the danger that sometimes a Lie 2-algebra of string type might be trivial, that is, the Jacobiator might correspond to the trivial element in $H^{3}(\mathfrak{k}, \mathbb{Z})$. Then what we have can be trivially strictified to a strict Lie 2-algebra, which is a crossed module of Lie algebras. Then the integration of a crossed module of Lie algebras is simply a crossed module of Lie groups. However, we will verify that the example we consider is not such a case.

The standard Courant algebroid motivates us to consider the case of the direct sum $\mathfrak{k}=\mathfrak{g} \oplus \mathfrak{g}^{*}$ of a Lie algebra $\mathfrak{g}$ and its dual with the semidirect product Lie algebra structure:

$$
[X+\xi, Y+\eta]=[X, Y]_{\mathfrak{g}}+\operatorname{ad}_{X}^{*} \eta-\operatorname{ad}_{Y}^{*} \xi,
$$

where $[\cdot, \cdot]_{\mathfrak{g}}$ is the Lie bracket of $\mathfrak{g}$. The nondegenerate invariant pairing $\langle\cdot, \cdot\rangle$ on $\mathfrak{g} \oplus \mathfrak{g}^{*}$ is given by

$$
\langle X+\xi, Y+\eta\rangle=\frac{1}{2}(\eta(X)+\xi(Y)) \quad \text { for all } X+\xi, Y+\eta \in \mathfrak{g} \oplus \mathfrak{g}^{*} .
$$

With these definitions, $\left(\mathfrak{g} \oplus \mathfrak{g}^{*},[\cdot, \cdot],\langle\cdot, \cdot\rangle\right)$ is a quadratic Lie algebra. In fact, we have

$$
\begin{aligned}
\left\langle\left[X_{1}+\xi_{1}, X_{2}+\xi_{2}\right], X_{3}+\xi_{3}\right\rangle & =\left\langle\left[X_{1}, X_{2}\right]_{\mathfrak{g}}+\operatorname{ad}_{X}^{*} \xi_{2}-\operatorname{ad}_{Y}^{*} \xi_{1}, X_{3}+\xi_{3}\right\rangle \\
& =\left\langle\left[X_{1}, X_{2}\right]_{\mathfrak{g}}, \xi_{3}\right\rangle+\text { c.p. }
\end{aligned}
$$

Similarly, we have

$$
\left\langle X_{2}+\xi_{2},\left[X_{1}+\xi_{1}, X_{3}+\xi_{3}\right]\right\rangle=\left\langle\left[X_{1}, X_{3}\right]_{\mathfrak{g}}, \xi_{2}\right\rangle+\text { c.p. },
$$

which implies $\left\langle X_{2}+\xi_{2},\left[X_{1}+\xi_{1}, X_{3}+\xi_{3}\right]\right\rangle+\left\langle\left[X_{1}+\xi_{1}, X_{2}+\xi_{2}\right], X_{3}+\xi_{3}\right\rangle=0$, that is, the nondegenerate inner product $\langle\cdot, \cdot\rangle$ is invariant under the adjoint action. This example is a special case of [Lu and Weinstein 1990, Theorem 1.12] with $\mathfrak{g}^{*}$ equipped with the 0 Lie bracket. Thus $\left(\mathfrak{g}, \mathfrak{g}^{*}\right)$ forms a Lie bialgebra or equivalently $\left(\mathfrak{g} \oplus \mathfrak{g}^{*}, \mathfrak{g}, \mathfrak{g}^{*}\right)$ is a Manin triple. However, honestly we have not found other Lie bialgebras (Manin triples) giving rise to Lie 2-algebras of the form of semidirect products. 
We denote by

$$
\mathbb{R} \stackrel{0}{\longrightarrow} \mathfrak{g} \oplus \mathfrak{g}^{*} .
$$

the corresponding Lie 2 -algebra of string type of $\left(\mathfrak{g} \oplus \mathfrak{g}^{*},[\cdot, \cdot],\langle\cdot, \cdot\rangle\right)$. We denote by $\tilde{v}: \bigwedge^{3}\left(\mathfrak{g} \oplus \mathfrak{g}^{*}\right) \rightarrow \mathbb{R}$ the corresponding 3-cocycle (see (18))

$$
\begin{aligned}
\tilde{v}\left(X_{1}+\xi_{1}, X_{2}+\xi_{2}, X_{3}+\xi_{3}\right) & =\left\langle\left[X_{1}+\xi_{1}, X_{2}+\xi_{2}\right], X_{3}+\xi_{3}\right\rangle \\
& =\left\langle\left[X_{1}, X_{2}\right]_{\mathfrak{g}}, \xi_{3}\right\rangle+\text { c.p. }
\end{aligned}
$$

Proposition 2.12. $\left(\mathbb{R} \stackrel{0}{\rightarrow} \mathfrak{g}^{*}, \mu_{1}=0, \mu_{0}=\mathrm{ad}^{*}, v=[\cdot, \cdot]_{\mathfrak{g}}\right)$ is a 2-term representation up to homotopy of the Lie algebra $\mathfrak{g}$. Moreover the Lie 2-algebra of string type $\mathbb{R} \stackrel{0}{\longrightarrow} \mathfrak{g} \oplus \mathfrak{g}^{*}$ is the semidirect product of $\mathfrak{g}$ and the complex $\mathbb{R} \stackrel{0}{\longrightarrow} \mathfrak{g}^{*}$.

Proof. Since $\mathrm{d}=0$, we only need to verify that $\mu_{0}$ and $\mu_{1}$ are Lie algebra morphisms and (6). Both ad* $: \mathfrak{g} \rightarrow \operatorname{End}\left(\mathfrak{g}^{*}\right)$ and 0 are Lie algebra morphisms, and (6) follows from Jacobi identity of $[\cdot, \cdot]_{\mathfrak{g}}$.

Then it is not hard to see that the Lie 2-algebra of string type $\mathbb{R} \stackrel{0}{\rightarrow} \mathfrak{g} \oplus \mathfrak{g}^{*}$ with formulas in Definition 2.10 is exactly the semidirect product of $\mathfrak{g}$ with the complex $\left(\mathbb{R} \stackrel{0}{\longrightarrow} \mathfrak{g}^{*}, \mu_{1}=0, \mu_{0}=\mathrm{ad}^{*}, v=[\cdot, \cdot]_{\mathfrak{g}}\right)$ with the formulas (7) and (8).

Proposition 2.13. If the Lie algebra $\mathfrak{g}$ is semisimple, the Lie algebra 3-cocycle $\tilde{v}$ given by (20) is not exact.

Proof. Let $\langle\cdot, \cdot\rangle_{k}$ be the Killing form on $\mathfrak{g}$. The proof follows from the fact that the Cartan 3-form $\langle[\cdot, \cdot], \cdot\rangle_{k}$ on a semisimple Lie algebra is not exact. Since $\mathfrak{g}$ is semisimple, the Killing form $\langle\cdot, \cdot\rangle_{k}$ is nondegenerate. Identify $\mathfrak{g}^{*}$ and $\mathfrak{g}$ by using the Killing form $\langle\cdot, \cdot\rangle_{k}$ and let $\mathscr{Y}$ be the corresponding isomorphism,

$$
\langle\mathscr{K}(\xi), X\rangle_{k}=\langle\xi, X\rangle .
$$

Assume that $\tilde{v}=d \phi$ for some $\phi: \bigwedge^{2}\left(\mathfrak{g} \oplus \mathfrak{g}^{*}\right) \rightarrow \mathbb{R}$; define $\varphi: \bigwedge^{2}(\mathfrak{g} \oplus \mathfrak{g}) \rightarrow \mathbb{R}$ by

$$
\phi(X+\xi, Y+\eta)=\varphi(X+\mathscr{K}(\xi), Y+\mathscr{K}(\eta)) .
$$

Then we have

$$
\begin{aligned}
\tilde{v}(X, Y, \xi) & =d \phi(X, Y, \xi) \\
& =-\phi([X, Y], \xi)+\phi\left(\operatorname{ad}_{X}^{*} \xi, Y\right)-\phi\left(\operatorname{ad}_{Y}^{*} \xi, X\right) \\
& =-\varphi([X, Y], \mathscr{K}(\xi))+\varphi([X, \mathscr{K}(\xi)], Y)-\varphi([Y, \mathscr{K}(\xi)], X) \\
& =d \varphi(X, Y, \mathscr{K}(\xi)) .
\end{aligned}
$$

On the other hand, we have

$$
\tilde{v}(X, Y, \xi)=\langle[X, Y], \xi\rangle=\langle[X, Y], \mathscr{K}(\xi)\rangle_{k},
$$


which implies that the Cartan 3-form

$$
\langle[X, Y], \mathscr{K}(\xi)\rangle_{k}=d \varphi(X, Y, \mathscr{K}(\xi))
$$

is exact. This is a contradiction.

In Section 4, we will give the integration of the Lie 2-algebra of string type $\mathbb{R} \stackrel{0}{\longrightarrow} \mathfrak{g} \oplus \mathfrak{g}^{*}$ by using the semidirect product of a Lie group with its 2 -term representation up to homotopy. It turns out that this Lie 2-algebra of string type can be integrated to a special Lie 2-group with a finite-dimensional model.

Example III (Omni-Lie algebroids $\mathfrak{D} E \oplus \mathfrak{J} E$ ). Chen and Liu [2010] introduced omni-Lie algebroids to generalize Weinstein's omni-Lie algebras. Just as Dirac structures of an omni-Lie algebra characterize Lie algebra structures on a vector space, Dirac structures of an omni-Lie algebroid characterize Lie algebroid structures on a vector bundle. See [Chen et al. 2008] for more details. In fact, the role of the omni-Lie algebroids $\mathfrak{D} E \oplus \mathfrak{J} E$ in $E$-Courant algebroids, which were introduced in [Chen et al. 2010], is the same as the role of standard Courant algebroids $T M \oplus T^{*} M$ in Courant algebroids.

We briefly recall the notion of omni-Lie algebroids. We will see that it gives rise to a 2-term $L_{\infty}$-algebra that is a semidirect product. In this subsection $E$ is a vector bundle over a smooth manifold $M$, and $\Gamma(E)$ is the section space of $E$.

Let $\mathfrak{D} E$ be the covariant differential operator bundle of a vector bundle $E$. The associated Atiyah sequence is given by

$$
0 \rightarrow \mathfrak{g l}(E) \stackrel{\dot{\mathrm{i}}}{\longrightarrow} \mathfrak{D} E \stackrel{a}{\longrightarrow} T M \rightarrow 0 .
$$

We define the associated 1 -jet vector bundle $\mathfrak{J} E$ as follows. For any $m \in M$, we define $(\mathfrak{J} E)_{m}$ as a quotient of local sections of $E$. Two local sections $u_{1}$ and $u_{2}$ are equivalent (we denote this by $u_{1} \sim u_{2}$ ) if

$$
u_{1}(m)=u_{2}(m) \quad \text { and } \quad d\left\langle u_{1}, \xi\right\rangle_{m}=d\left\langle u_{2}, \xi\right\rangle_{m} \quad \text { for all } \xi \in \Gamma\left(E^{*}\right) .
$$

So any $\mu \in(\mathfrak{J} E)_{m}$ has a representative $u \in \Gamma(E)$ such that $\mu=[u]_{m}$. Let $\mathbb{p}$ be the projection that sends $[u]_{m}$ to $u(m)$. Then $\operatorname{Ker} \mathbb{p} \cong \operatorname{Hom}(T M, E)$ and there is a short exact sequence

$$
0 \rightarrow \operatorname{Hom}(T M, E) \stackrel{\mathbb{e}}{\longrightarrow} \mathfrak{J} E \stackrel{\mathrm{p}}{\longrightarrow} E \rightarrow 0,
$$

called the jet sequence of $E$. From this it is straightforward to see that $\mathfrak{J} E$ is a finite dimensional vector bundle. Also, $\Gamma(\mathfrak{J} E)$ is isomorphic to $\Gamma(E) \oplus \Gamma\left(T^{*} M \otimes E\right)$ as an $\mathbb{R}$-vector space, and any $u \in \Gamma(E)$ has a lift $\mathbb{d} u \in \Gamma(\mathfrak{J} E)$ by taking its equivalence class, such that

$$
\mathbb{d}(f u)=f \mathbb{d} u+d f \otimes u \quad \text { for all } f \in C^{\infty}(M) .
$$


Chen and Liu [2010] proved that

$$
\mathfrak{J} E \cong\left\{v \in \operatorname{Hom}(\mathfrak{D} E, E) \mid v(\Phi)=\Phi \circ v\left(\operatorname{Id}_{E}\right) \text { for all } \Phi \in \mathfrak{g l}(E)\right\} .
$$

Therefore, there is an $E$-pairing between $\mathfrak{J} E$ and $\mathfrak{D} E$ obtained by setting

$$
\langle\mu, \mathfrak{d}\rangle_{E} \triangleq \mathfrak{d}(u) \quad \text { for all } \mu \in(\mathfrak{J} E)_{m}, \mathfrak{d} \in(\mathfrak{D} E)_{m},
$$

where $u \in \Gamma(E)$ satisfies $\mu=[u]_{m}$. Particularly, one has

$$
\begin{aligned}
\langle\mu, \Phi\rangle_{E} & =\Phi \circ \mathfrak{p}(\mu) & & \text { for all } \Phi \in \mathfrak{g l}(E), \mu \in \mathfrak{J} E ; \\
\langle\mathfrak{y}, \mathfrak{d}\rangle_{E} & =\mathfrak{y} \circ a(\mathfrak{d}) & & \text { for all } \mathfrak{y} \in \operatorname{Hom}(T M, E), \mathfrak{d} \in \mathfrak{D} E .
\end{aligned}
$$

Furthermore, we claim that $\Gamma(\mathfrak{J} E)$ is an invariant subspace of the Lie derivative $\mathfrak{L}_{\mathfrak{d}}$ for any $\mathfrak{d} \in \Gamma(\mathfrak{D} E)$, which is defined by the Leibniz rule as follows:

$$
\left\langle\mathfrak{L}_{\mathfrak{d}} \mu, \mathfrak{d}^{\prime}\right\rangle_{E} \triangleq \mathfrak{d}\left\langle\mu, \mathfrak{d}^{\prime}\right\rangle_{E}-\left\langle\mu,\left[\mathfrak{d}, \mathfrak{d}^{\prime}\right]_{\mathfrak{D}}\right\rangle_{E} \quad \text { for all } \mu \in \Gamma(\mathfrak{J} E), \mathfrak{d}^{\prime} \in \Gamma(\mathfrak{D} E) .
$$

Define a nondegenerate symmetric $E$-valued 2-form $(\cdot, \cdot)_{E}$ on $\mathscr{E} \triangleq \mathfrak{D} E \oplus \mathfrak{J} E$ by

$$
(\mathfrak{d}+\mu, \mathfrak{r}+v)_{E} \triangleq \frac{1}{2}\left(\langle\mathfrak{d}, v\rangle_{E}+\langle\mathfrak{r}, \mu\rangle_{E}\right) \quad \text { for all } \mathfrak{d}, \mathfrak{r} \in \mathfrak{D} E, \mu, v \in \mathfrak{J} E .
$$

Define an antisymmetric bracket $\llbracket \cdot, \cdot \rrbracket$ on $\Gamma(\mathscr{E})$ by

$$
\llbracket \mathfrak{d}+\mu, \mathfrak{r}+v \rrbracket \triangleq[\mathfrak{d}, \mathfrak{r}]_{\mathfrak{D}}+\mathfrak{L}_{\mathfrak{d}} v-\mathfrak{L}_{\mathfrak{r}} \mu+\frac{1}{2}\left(\mathbb{d}\langle\mu, \mathfrak{r}\rangle_{E}-\mathbb{d}\langle v, \mathfrak{d}\rangle_{E}\right) .
$$

Chen and Liu [2010] call the quadruple $\left(\mathscr{E}, \mathbb{[} \cdot, \cdot \rrbracket,(\cdot, \cdot)_{E}, \rho_{\varepsilon}\right)$ the omni-Lie algebroid associated to the vector bundle $E$, where $\rho$ is the projection of $\mathscr{E}$ onto $\mathfrak{D} E .^{3}$ Even though $\llbracket \cdot, \cdot \rrbracket$ is antisymmetric, it is not a Lie bracket. More precisely, we have

$$
\llbracket \llbracket X, Y \rrbracket, Z \rrbracket+\text { c.p. }=\mathbb{d} T(X, Y, Z) \quad \text { for any } X, Y, Z \in \Gamma(\mathscr{E}),
$$

where $T: \Gamma\left(\bigwedge^{3} \mathscr{E}\right) \rightarrow \Gamma(E)$ is defined by

$$
T(X, Y, Z)=\frac{1}{3}\left((\llbracket X, Y \rrbracket, Z)_{E}+\text { c.p. }\right) .
$$

Let us construct a 2 -term $L_{\infty}$-algebra from the omni-Lie algebroid $\mathscr{E}$. Obviously, $\Gamma(\mathfrak{D} E)$ is a Lie algebra and there is a natural 2-term complex

$$
\Gamma(E) \stackrel{0 \oplus \mathbb{d}}{\longrightarrow} \Gamma(\mathfrak{J} E) .
$$

For any $\mathfrak{d} \in \Gamma(\mathfrak{D} E)$, define linear actions $\mu_{0}$ and $\mu_{1}$ by

$$
\begin{array}{ll}
\mu_{0}(\mathfrak{d})(\mu) \triangleq \llbracket \mathfrak{d}, \mu \rrbracket=\mathfrak{L}_{\mathfrak{d}} \mu-\mathbb{d}(\mu, \mathfrak{d})_{E} & \text { for all } \mu \in \Gamma(\mathfrak{J} E), \\
\mu_{1}(\mathfrak{d})(u) \triangleq(\mathfrak{d}, \mathbb{d} u)_{E}=\frac{1}{2} \mathfrak{d}(u) & \text { for all } u \in \Gamma(E) .
\end{array}
$$

${ }^{3}$ This is slightly different from the notion given in [Chen and Liu 2010], where the bracket is not skew symmetric. 
Define $v: \bigwedge^{2} \Gamma(\mathfrak{D} E) \rightarrow \operatorname{Hom}(\Gamma(\mathfrak{J} E), \Gamma(E))$ by

$$
v(\mathfrak{d}, \mathfrak{t})(\xi)=T(\mathfrak{d}, \mathfrak{t}, \mu) \quad \text { for all } \mathfrak{d}, \mathfrak{t} \in \Gamma(\mathfrak{D} E), \mu \in \Gamma(\mathfrak{J} E) .
$$

Similarly to Proposition 2.6, we prove:

Proposition 2.14. With the notations above, $\left(\Gamma(E) \stackrel{0 \oplus \mathbb{d}}{\longrightarrow} \Gamma(\mathfrak{J} E), \mu=\mu_{0}+\mu_{1}, v\right)$ is a representation up to homotopy of the Lie algebra $\Gamma(\mathfrak{D} E)$.

Corollary 2.15. $\left(\Gamma(E) \stackrel{0 \oplus \mathbb{d}}{\longrightarrow} \Gamma(\mathfrak{D} E) \oplus \Gamma(\mathfrak{J} E), l_{2}, l_{3}\right)$ is a 2-term $L_{\infty}$-algebra, where $l_{2}$ and $l_{3}$ are given by (7) and (8), and $\mu$ and $v$ are given by (28) and (29), respectively.

Remark 2.16. If the base manifold $M$ is a point, that is, $E$ is a vector space, for which we use a new notation $V$, then $\mathfrak{D} V=\mathfrak{g l}(V)$ and $\mathfrak{J} V=V$, and we recover the notion of omni-Lie algebras. The complex $\Gamma(V) \stackrel{0 \oplus \mathbb{d}}{\longrightarrow} \Gamma(\mathfrak{J} V)$ reduces to $V \stackrel{\mathrm{Id}}{\longrightarrow} V$, which is a representation up to homotopy of $\mathfrak{g l}(V)$ with $\left(\mu_{0}=\mu_{1}, v\right)$ given by

$$
\mu_{0}(A)(u)=\frac{1}{2} A u, \quad v(A, B)=\frac{1}{4}[A, B] \quad \text { for all } A, B \in \mathfrak{g l}(V), u \in V .
$$

Hence even though an omni-Lie algebra $\mathfrak{g l}(V) \oplus V$ is not a Lie algebra, we can extend it to a 2-term $L_{\infty}$-algebra, of which $L_{0}=\mathfrak{g l}(V) \oplus V, L_{1}=V, l_{2}$ and $l_{3}$ are given by (7) and (8), in which $\mu$ and $v$ are given by (30). This 2-term $L_{\infty}$-algebra is a semidirect product of $\mathfrak{g l}(V)$ with $V \stackrel{\mathrm{Id}}{\longrightarrow} V$.

We will study the global object of the 2-term $L_{\infty}$-algebra associated to an omniLie algebra in the forthcoming paper [Sheng and Zhu 2010].

\section{Representations up to homotopy of Lie groups and semidirect products}

The representation up to homotopy of a Lie group was introduced in [Abad 2008]. In this section we define the semidirect product of a Lie group with a 2-term representation up to homotopy and prove that the semidirect product is a Lie 2-group. Thus we first recall some background on Lie 2-groups.

A group is a monoid where every element has an inverse. A 2-group is a monoidal category where every object has a weak inverse and every morphism has an inverse. Denote the category of smooth manifolds and smooth maps by Diff, a semistrict ${ }^{4}$ Lie 2-group is a 2-group in DiffCat, where DiffCat is the 2-category consisting of categories, functors, and natural transformations in Diff. For more details, see [Baez and Lauda 2004]. Here we only recall the expanded definition:

Definition 3.1 [Baez and Lauda 2004]. A semistrict Lie 2-group consists of an object $C$ in DiffCat, that is,

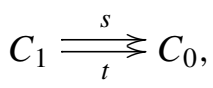

\footnotetext{
${ }^{4}$ See the introduction for the reason we call it a semistrict Lie 2-group.
} 
where $C_{1}$ and $C_{0}$ are objects in Diff, $s$ and $t$ are the source and target maps, and there is a vertical multiplication $\cdot_{\mathrm{v}}: C \times C \rightarrow C$, together with

- a functor (horizontal multiplication) ${ }_{\mathrm{h}}: C \times C \rightarrow C$,

- an identity object 1 ,

- a contravariant functor inv : $C \rightarrow C$

and the following natural isomorphisms:

- the associator $a_{x, y, z}:(x \cdot \mathrm{h} y) \cdot_{\mathrm{h}} z \rightarrow x \cdot \mathrm{h}(y \cdot \mathrm{h} z)$,

- the left and right unit $l_{x}: 1 \cdot{ }_{\mathrm{h}} x \rightarrow x$ and $r_{x}: x \cdot{ }_{\mathrm{h}} 1 \rightarrow x$,

- the unit and counit $i_{x}: 1 \rightarrow x \cdot \mathrm{h} \operatorname{inv}(x)$ and $e_{x}: \operatorname{inv}(x) \cdot{ }_{\mathrm{h}} x \rightarrow 1$,

which are such that the following diagrams commute.

- The pentagon identity for the associator:

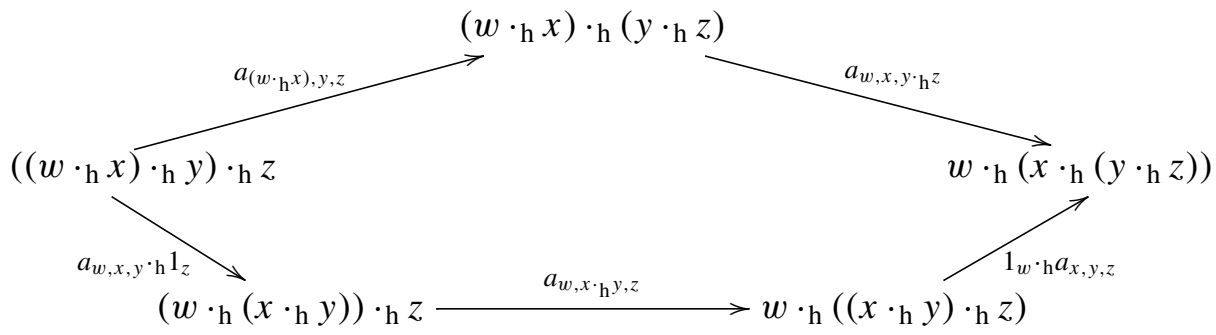

- The triangle identity for the left and right unit lows:

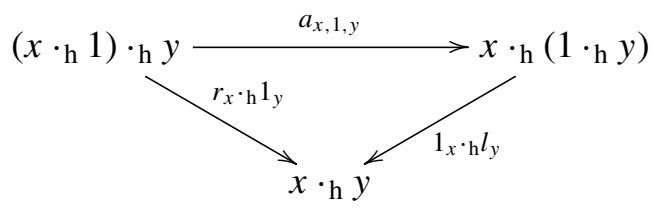

- The first zig-zag identity:

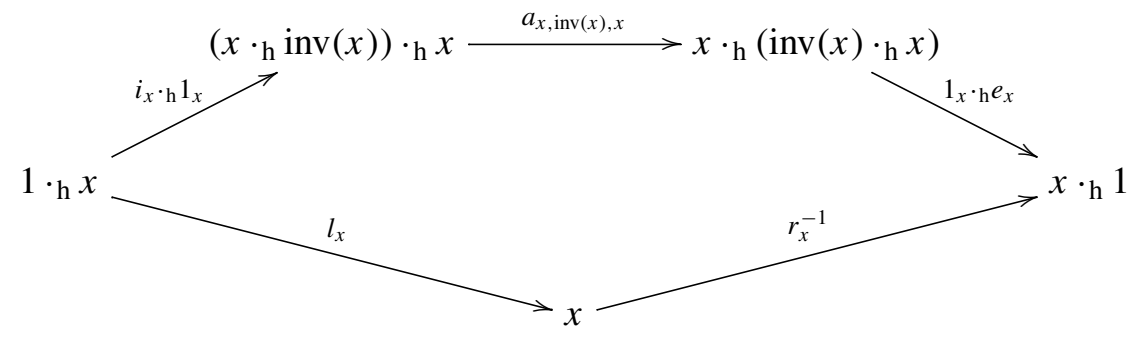


- The second zig-zag identity:

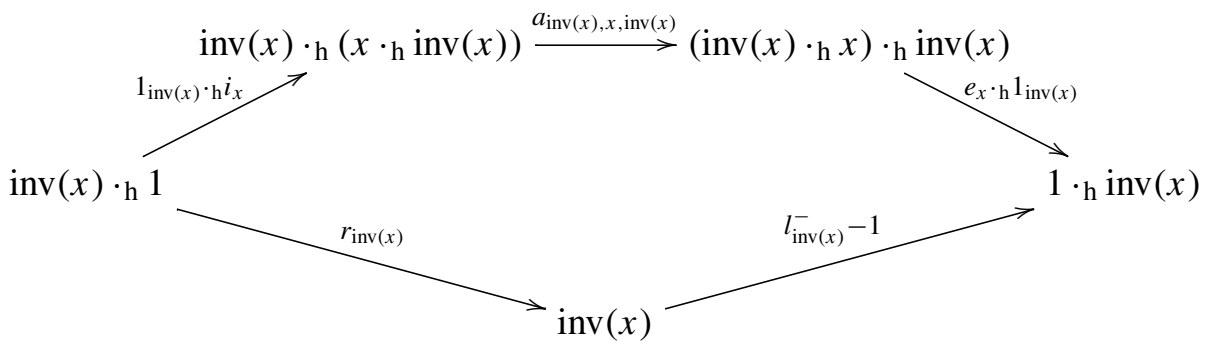

In the special case where $a_{x, y, z}, l_{x}, r_{x}, i_{x}$ and $e_{x}$ are all identity isomorphisms, we call such a Lie 2-group a strict Lie 2-group. ${ }^{5}$

Definition 3.2 [Baez and Lauda 2004]. A special Lie 2-group is a Lie 2-group of which the source and target coincide and the left unit law $l$, the right unit law $r$, the unit $i$ and the counit $e$ are identity isomorphisms.

For classification of special Lie 2-groups, we need the group cohomology with smooth cocycles, that is, we consider the cochain complex with smooth morphisms $G^{\times n} \rightarrow M$ with $G$ a Lie group and $M$ its module. The differential is defined as usual for group cohomology. We denote this cohomology by $H_{\mathrm{sm}}^{\bullet}(G, M)$.

Theorem 3.3 [Baez and Lauda 2004, Theorem 8.3.7]. There is a one-to-one correspondence between special Lie 2-groups and quadruples $\left(K_{1}, K_{2}, \Phi, \Theta\right)$ consisting a Lie group $K_{1}$, an abelian group $K_{2}$, an action $\Phi$ of $K_{1}$ as automorphisms of $K_{2}$ and a normalized smooth 3-cocycle $\Theta: K_{1}^{3} \rightarrow K_{2}$. Two special Lie 2-groups are isomorphic if and only if they correspond to the same $e^{6}\left(K_{1}, K_{2}, \Phi\right)$ and the corresponding 3-cocycles represent the same element in $H_{\mathrm{sm}}^{3}\left(K_{1}, K_{2}\right)$.

Remark 3.4. Given a quadruple $\left(K_{1}, K_{2}, \Phi, \Theta\right)$, the corresponding semistrict Lie 2-group has the Lie group $K_{1}$ as the space of objects and the semidirect product Lie group $K_{1} \ltimes_{\Phi} K_{2}$ as the space of morphisms. The associator is given by $\Theta$.

Definition 3.5. A unital 2-term representation up to homotopy of a Lie group $G$ consists of

(a) a 2-term complex of vector spaces $V_{1} \stackrel{\text { d }}{\rightarrow} V_{0}$;

(b) a nonassociative action $F_{1}$ on $V_{0}$ and $V_{1}$ satisfying $\mathrm{d} F_{1}=F_{1} \mathrm{~d}$ and $F_{1}\left(1_{G}\right)=\mathrm{Id}$; and

(c) a smooth map $F_{2}: G \times G \rightarrow \operatorname{End}\left(V_{0}, V_{1}\right)$ such that

$$
F_{1}\left(g_{1}\right) \cdot F_{1}\left(g_{2}\right)-F_{1}\left(g_{1} \cdot g_{2}\right)=\left[\mathrm{d}, F_{2}\left(g_{1}, g_{2}\right)\right]
$$

\footnotetext{
${ }^{5}$ The notion of strict Lie 2-groups is the same as in [Baez and Lauda 2004].

${ }^{6}$ up to isomorphisms of groups, of course
} 
and

(32)

$F_{1}\left(g_{1}\right) \circ F_{2}\left(g_{2}, g_{3}\right)-F_{2}\left(g_{1} \cdot g_{2}, g_{3}\right)+F_{2}\left(g_{1}, g_{2} \cdot g_{3}\right)-F_{2}\left(g_{1}, g_{2}\right) \circ F_{1}\left(g_{3}\right)=0$.

We denote this 2-term representation up to homotopy of the Lie group $G$ by $\left(V_{1} \stackrel{\mathrm{d}}{\rightarrow} V_{0}, F_{1}, F_{2}\right)$. One should be careful: Even if $F_{1}$ is a usual associative action, (32) is not equivalent to $F_{2}$ being a 2-cocycle. This is strangely different from the Lie algebra case (see Section 4$)$. Define $\widetilde{F}_{2}:\left(G \ltimes V_{0}\right)^{3} \rightarrow V_{1}$ by

$$
\widetilde{F}_{2}\left(\left(g_{1}, \xi_{1}\right),\left(g_{2}, \xi_{2}\right),\left(g_{3}, \xi_{3}\right)\right)=F_{2}\left(g_{1}, g_{2}\right)\left(\xi_{3}\right) .
$$

If $F_{1}$ is a usual associative action, we form the semidirect product $G \ltimes V_{0}$. Then $V_{1}$ is a $G \ltimes V_{0}$-module with an associated action $\widetilde{F}_{1}$ of $G \ltimes V_{0}$ on $V_{1}$ given by

$$
\widetilde{F}_{1}(g, \xi)(m)=F_{1}(g)(m) \text { for all } m \in V_{1} .
$$

Proposition 3.6. If $F_{1}$ is the usual associative action of the Lie group $G$ on the complex $V_{1} \stackrel{\mathrm{d}}{\longrightarrow} V_{0}$, then $\widetilde{F}_{2}$ defined by (33) is a group 3-cocycle representing an element in $H_{\mathrm{sm}}^{3}\left(G \ltimes V_{0}, V_{1}\right)$.

Proof. By direct computation, we have

$$
\begin{aligned}
d & \widetilde{F}_{2}\left(\left(g_{1}, \xi_{1}\right),\left(g_{2}, \xi_{2}\right),\left(g_{3}, \xi_{3}\right),\left(g_{4}, \xi_{4}\right)\right) \\
= & \widetilde{F}_{1}\left(g_{1}, \xi_{1}\right) \widetilde{F}_{2}\left(\left(g_{2}, \xi_{2}\right),\left(g_{3}, \xi_{3}\right),\left(g_{4}, \xi_{4}\right)\right) \\
& -\widetilde{F}_{2}\left(\left(g_{1}, \xi_{1}\right) \cdot\left(g_{2}, \xi_{2}\right),\left(g_{3}, \xi_{3}\right),\left(g_{4}, \xi_{4}\right)\right)+\widetilde{F}_{2}\left(\left(g_{1}, \xi_{1}\right),\left(g_{2}, \xi_{2}\right) \cdot\left(g_{3}, \xi_{3}\right),\left(g_{4}, \xi_{4}\right)\right) \\
& -\widetilde{F}_{2}\left(\left(g_{1}, \xi_{1}\right),\left(g_{2}, \xi_{2}\right),\left(g_{3}, \xi_{3}\right) \cdot\left(g_{4}, \xi_{4}\right)\right)+\widetilde{F}_{2}\left(\left(g_{1}, \xi_{1}\right),\left(g_{2}, \xi_{2}\right),\left(g_{3}, \xi_{3}\right)\right) \\
= & F_{1}\left(g_{1}\right) F_{2}\left(g_{2}, g_{3}\right)\left(\xi_{4}\right)-F_{2}\left(g_{1} \cdot g_{2}, g_{3}\right)\left(\xi_{4}\right)+F_{2}\left(g_{1}, g_{2} \cdot g_{3}\right)\left(\xi_{4}\right) \\
& -F_{2}\left(g_{1}, g_{2}\right)\left(\xi_{3}+F_{1}\left(g_{3}\right)\left(\xi_{4}\right)\right)+F_{2}\left(g_{1}, g_{2}\right)\left(\xi_{3}\right) \\
= & \left(F_{1}\left(g_{1}\right) \circ F_{2}\left(g_{2}, g_{3}\right)-F_{2}\left(g_{1} \cdot g_{2}, g_{3}\right)+F_{2}\left(g_{1}, g_{2} \cdot g_{3}\right)-F_{2}\left(g_{1}, g_{2}\right) \circ F_{1}\left(g_{3}\right)\right)\left(\xi_{4}\right) .
\end{aligned}
$$

By (32), $\widetilde{F}_{2}$ is a Lie group 3-cocycle.

Just as we can associate to any representation of a Lie group a new Lie group that is their semidirect product, we can use a 2-term representation up to homotopy of a Lie group to form a Lie 2-group.

Theorem 3.7. Given a 2-term representation up to homotopy $\left(V_{1} \stackrel{\mathrm{d}}{\rightarrow} V_{0}, F_{1}, F_{2}\right)$ of a Lie group $G$, its semidirect product with $G$ is defined to be

$$
\begin{gathered}
G \times V_{0} \times V_{1} \\
s \| t \\
\quad \downarrow^{t} \\
G \times V_{0} .
\end{gathered}
$$

Then it is a Lie 2-group with the following structure maps: 
The source and target are given by

$$
s(g, \xi, m)=(g, \xi) \text { and } t(g, \xi, m)=(g, \xi+\mathrm{d} m) .
$$

The vertical multiplication $\cdot_{\mathrm{v}}$ is given by

$$
(h, \eta, n) \cdot{ }_{\mathrm{v}}(g, \xi, m)=(g, \xi, m+n), \quad \text { where } h=g, \eta=\xi+\mathrm{d} m .
$$

The horizontal multiplication $\cdot \mathrm{h}$ of objects is given by

$$
\left(g_{1}, \xi\right) \cdot{ }_{h}\left(g_{2}, \eta\right)=\left(g_{1} \cdot g_{2}, \xi+F_{1}\left(g_{1}\right)(\eta)\right)
$$

The horizontal multiplication ${ }_{\mathrm{h}} \mathrm{o}$ of morphisms is given by

$$
\left(g_{1}, \xi, m\right) \cdot_{\mathrm{h}}\left(g_{2}, \eta, n\right)=\left(g_{1} \cdot g_{2}, \xi+F_{1}\left(g_{1}\right)(\eta), m+F_{1}\left(g_{1}\right)(n)\right) .
$$

The inverse map inv is given by

$$
\operatorname{inv}(g, \xi)=\left(g^{-1},-F_{1}\left(g^{-1}\right)(\xi)\right) .
$$

The identity object is $\left(1_{G}, 0\right)$.

The associator

$$
a_{\left(g_{1}, \xi\right),\left(g_{2}, \eta\right),\left(g_{3}, \gamma\right)}:\left(\left(g_{1}, \xi\right) \cdot{ }_{\mathrm{h}}\left(g_{2}, \eta\right)\right) \cdot{ }_{\mathrm{h}}\left(g_{3}, \gamma\right) \rightarrow\left(g_{1}, \xi\right) \cdot{ }_{\mathrm{h}}\left(\left(g_{2}, \eta\right) \cdot{ }_{\mathrm{h}}\left(g_{3}, \gamma\right)\right)
$$

is given by

(39) $a_{\left(g_{1}, \xi\right),\left(g_{2}, \eta\right),\left(g_{3}, \gamma\right)}=\left(g_{1} \cdot g_{2} \cdot g_{3}, \xi+F_{1}\left(g_{1}\right)(\eta)+F_{1}\left(g_{1} \cdot g_{2}\right)(\gamma), F_{2}\left(g_{1}, g_{2}\right)(\gamma)\right)$.

The unit $i_{(g, \xi)}:\left(1_{G}, 0\right) \rightarrow(g, \xi) \cdot{ }_{h} \operatorname{inv}(g, \xi)$ is given by

$$
i_{(g, \xi)}=\left(1_{G}, 0,-F_{2}\left(g, g^{-1}\right)(\xi)\right) .
$$

All the other natural isomorphisms are identity isomorphisms.

Proof. By (35), (36) and (37), it is straightforward to see that

$$
\begin{aligned}
& s\left(\left(g_{1}, \xi, m\right) \cdot{ }_{\mathrm{h}}\left(g_{2}, \eta, n\right)\right)=s\left(g_{1}, \xi, m\right) \cdot_{\mathrm{h}} s\left(g_{2}, \eta, n\right), \\
& t\left(\left(g_{1}, \xi, m\right) \cdot{ }_{\mathrm{h}}\left(g_{2}, \eta, n\right)\right)=t\left(g_{1}, \xi, m\right) \cdot{ }_{\mathrm{h}} t\left(g_{2}, \eta, n\right) .
\end{aligned}
$$

Thus the multiplication ${ }^{h} \mathrm{~h}$ respects the source and target map. Furthermore, it is not hard to check that the horizontal and vertical multiplications commute, that is,

$$
\begin{aligned}
\left((g, \xi+\mathrm{d} m, n) \cdot{ }_{\mathrm{h}}\left(g^{\prime}, \eta+\mathrm{d} p, q\right)\right) \cdot \cdot_{\mathrm{v}}\left((g, \xi, m) \cdot{ }_{\mathrm{h}}\left(g^{\prime}, \eta, p\right)\right) \\
\quad=\left((g, \xi+\mathrm{d} m, n) \cdot \cdot_{\mathrm{v}}(g, \xi, m)\right) \cdot{ }_{\mathrm{h}}\left(\left(g^{\prime}, \eta+\mathrm{d} p, q\right) \cdot \cdot_{\mathrm{v}}\left(g^{\prime}, \eta, p\right)\right)
\end{aligned}
$$


or, in terms of a diagram,

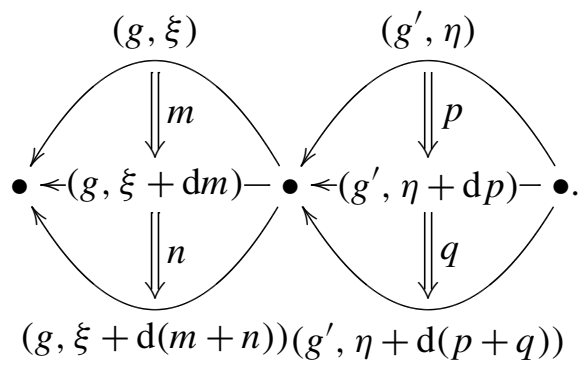

It follows from (31) that the associator $a_{\left(g_{1}, \xi\right),\left(g_{2}, \eta\right),\left(g_{3}, \gamma\right)}$ defined by (39) is indeed a morphism from $\left(\left(g_{1}, \xi\right) \cdot{ }_{\mathrm{h}}\left(g_{2}, \eta\right)\right) \cdot_{\mathrm{h}}\left(g_{3}, \gamma\right)$ to $\left(g_{1}, \xi\right) \cdot_{\mathrm{h}}\left(\left(g_{2}, \eta\right) \cdot_{\mathrm{h}}\left(g_{3}, \gamma\right)\right)$. To see that it is natural, we need to show that

$$
a_{\left(g_{1}, \xi+\mathrm{d} m\right),\left(g_{2}, \eta+\mathrm{d} n\right),\left(g_{3}, \gamma+\mathrm{d} k\right) \cdot \mathrm{h}}\left(\left(\left(g_{1}, \xi, m\right) \cdot_{\mathrm{h}}\left(g_{2}, \eta, n\right)\right) \cdot_{\mathrm{h}}\left(g_{3}, \gamma, k\right)\right)
$$

is equal to

$$
\left(\left(g_{1}, \xi, m\right) \cdot{ }_{\mathrm{h}}\left(\left(g_{2}, \eta, n\right) \cdot{ }_{\mathrm{h}}\left(g_{3}, \gamma, k\right)\right)\right) \cdot_{\mathrm{h}} a_{\left(g_{1}, \xi\right),\left(g_{2}, \eta\right),\left(g_{3}, \gamma\right),}
$$

that is, the following diagram commutes:

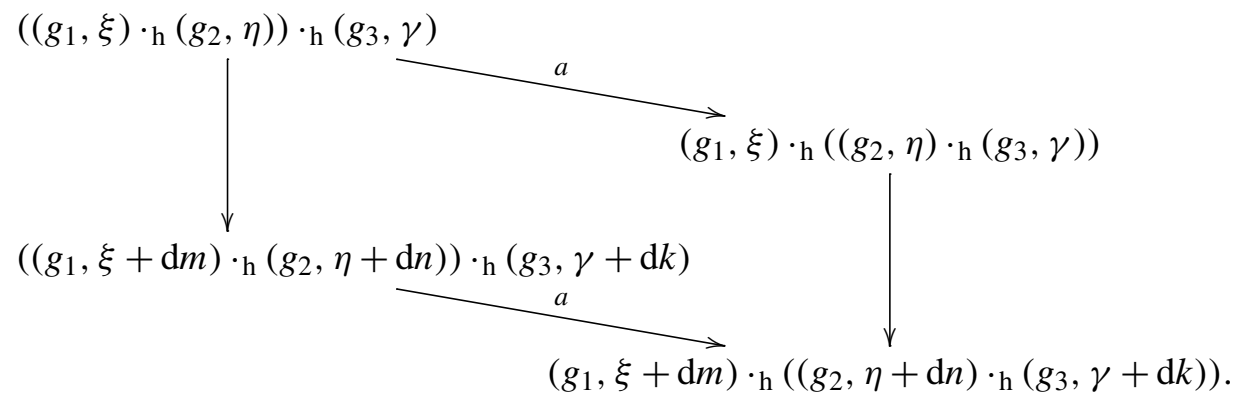

By straightforward computations, we obtain that (42) is equal to $\left(g_{1} \cdot g_{2} \cdot g_{3}\right.$, $\left.\xi+F_{1}\left(g_{1}\right)(\eta)+F_{1}\left(g_{1} \cdot g_{2}\right)(\gamma), m+F_{1}\left(g_{1}\right)(n)+F_{1}\left(g_{1} \cdot g_{2}\right)(k)+F_{2}\left(g_{1}, g_{2}\right)(\gamma+\mathrm{d} k)\right)$, and (43) is equal to

$\left(g_{1} \cdot g_{2} \cdot g_{3}\right.$, $\left.\xi+F_{1}\left(g_{1}\right)(\eta)+F_{1}\left(g_{1} \cdot g_{2}\right)(\gamma), m+F_{1}\left(g_{1}\right)(n)+F_{1}\left(g_{1}\right) \cdot F_{1}\left(g_{2}\right)(k)+F_{2}\left(g_{1}, g_{2}\right)(\gamma)\right)$.

Hence (42) is equal to (43) by (31). This implies that $a_{\left(g_{1}, \xi\right),\left(g_{2}, \eta\right),\left(g_{3}, \gamma\right)}$ as defined by (39) is a natural isomorphism. 
By (31) and the fact that $F_{1}\left(1_{G}\right)=\mathrm{Id}$, the unit given by (40) is indeed a morphism from $\left(1_{G}, 0\right)$ to $(g, \xi) \cdot_{h} \operatorname{inv}(g, \xi)$. To see that it is natural, we need to prove that

$$
\left((g, \xi, m) \cdot{ }_{\mathrm{h}} \operatorname{inv}(g, \xi, m)\right) \cdot{ }_{\mathrm{h}} i_{(g, \xi)}=i_{(g, \xi+\mathrm{d} m)},
$$

that is, that the diagram

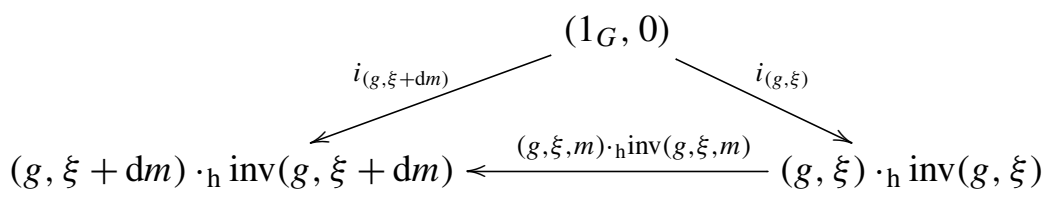

commutes. This follows from

$F_{2}\left(g, g^{-1}\right)(\mathrm{d} m)=F_{1}(g) \cdot F_{1}\left(g^{-1}\right)(m)-F_{1}\left(g \cdot g^{-1}\right)(m)=F_{1}(g) \cdot F_{1}\left(g^{-1}\right)(m)-m$,

which is a special case of (31).

Since $F\left(1_{G}\right)=\mathrm{Id}$, we have

$$
\left(1_{G}, 0\right) \cdot_{h}(g, \xi)=(g, \xi) \quad \text { and } \quad(g, \xi) \cdot_{h}\left(1_{G}, 0\right)=(g, \xi) .
$$

Hence the left unit and the right unit can also be taken as the identity isomorphism.

The counit $e_{(g, \xi)}: \operatorname{inv}(g, \xi) \cdot \mathrm{h}(g, \xi) \rightarrow\left(1_{G}, 0\right)$ can be taken as the identity morphism since

$$
\operatorname{inv}(g, \xi) \cdot \mathrm{h}(g, \xi)=\left(g^{-1},-F_{1}\left(g^{-1}\right)(\xi)\right) \cdot \mathrm{h}(g, \xi)=\left(1_{G}, 0\right) .
$$

Finally, we need to show the pentagon identity for the associator, the triangle identity for the left and right unit laws, and the first and second zig-zag identities. We only give the proof of the pentagon identity; we leave the similar proofs of the others to the reader. In fact, the pentagon identity is equivalent to

$a_{\left(g_{1}, \xi\right),\left(g_{2}, \eta\right),\left(g_{3}, \gamma\right) \cdot{ }_{\mathrm{h}}\left(g_{4}, \theta\right) \cdot{ }_{\mathrm{v}}} a_{\left(g_{1}, \xi\right) \cdot{ }_{\mathrm{h}}\left(g_{2}, \eta\right),\left(g_{3}, \gamma\right),\left(g_{4}, \theta\right)}$

$\left(\left(g_{1}, \xi\right) \cdot{ }_{\mathrm{h}} a_{\left(g_{2}, \eta\right),\left(g_{3}, \gamma\right),\left(g_{4}, \theta\right)}\right) \cdot{ }_{\mathrm{v}} a_{\left(g_{1}, \xi\right),\left(g_{2}, \eta\right) \cdot{ }_{\mathrm{h}}\left(g_{3}, \gamma\right),\left(g_{4}, \theta\right) \cdot{ }^{\mathrm{v}}}\left(a_{\left(g_{1}, \xi\right),\left(g_{2}, \eta\right),\left(g_{3}, \gamma\right) \cdot \mathrm{h}}\left(g_{4}, \theta\right)\right)$.

By straightforward computations, the left hand side is equal to

$$
\begin{aligned}
\left(g_{1} \cdot g_{2} \cdot g_{3} \cdot g_{4}, \xi+F_{1}\left(g_{1}\right)(\eta)+\right. & F_{1}\left(g_{1} \cdot g_{2}\right)(\gamma)+F_{1}\left(g_{1} \cdot g_{2} \cdot g_{3}\right)(\theta), \\
& \left.F_{2}\left(g_{1} \cdot g_{2}, g_{3}\right)(\theta)+F_{2}\left(g_{1}, g_{2}\right)\left(\gamma+F_{1}\left(g_{3}\right)(\theta)\right)\right),
\end{aligned}
$$

and the right hand side is equal to

$$
\begin{aligned}
\left(g_{1} \cdot g_{2} \cdot g_{3} \cdot g_{4}, \xi+\right. & F_{1}\left(g_{1}\right)(\eta)+F_{1}\left(g_{1} \cdot g_{2}\right)(\gamma)+F_{1}\left(g_{1} \cdot g_{2} \cdot g_{3}\right)(\theta), \\
& \left.F_{2}\left(g_{1}, g_{2}\right)(\gamma)+F_{2}\left(g_{1}, g_{2} \cdot g_{3}\right)(\theta)+F_{1}\left(g_{1}\right) \circ F_{2}\left(g_{2}, g_{3}\right)(\theta)\right) .
\end{aligned}
$$

By (32), they are equal. 


\section{Integrating the Lie 2-algebra of string type $\mathbb{R} \rightarrow \mathfrak{g} \oplus \mathfrak{g}^{*}$}

As an application of Theorem 3.7, we consider the integration of the Lie 2-algebra of string type $\mathbb{R} \rightarrow \mathfrak{g} \oplus \mathfrak{g}^{*}$ given by (19). Now we restrict to the case that $\mathfrak{g}$ is finite-dimensional. Obviously, given a quadruple $\left(K_{1}, K_{2}, \Phi, \Theta\right)$ that represents a special Lie 2-group (see Theorem 3.3), we obtain by differentiation a quadruple $\left(\mathfrak{k}_{1}, \mathfrak{k}_{2}, \phi, \theta\right)$, which represents a 2 -term skeletal $L_{\infty}$-algebra.

Definition 4.1. A special Lie 2-group that is represented by $\left(K_{1}, K_{2}, \Phi, \Theta\right)$ is an integration of a 2-term skeletal $L_{\infty}$-algebra that is represented by $\left(\mathfrak{k}_{1}, \mathfrak{k}_{2}, \phi, \theta\right)$ if the differentiation of $\left(K_{1}, K_{2}, \Phi, \Theta\right)$ is $\left(\mathfrak{k}_{1}, \mathfrak{k}_{2}, \phi, \theta\right)$.

If the differential $\mathrm{d}$ in a 2-term complex $V_{1} \stackrel{\mathrm{d}}{\rightarrow} V_{0}$ is 0 , a representation up to homotopy of Lie algebra $\mathfrak{g}$ on $V_{1} \stackrel{0}{\longrightarrow} V_{0}$ consists of two strict representations $\mu_{1}$ and $\mu_{0}$, and a linear map $v: \mathfrak{g} \wedge \mathfrak{g} \rightarrow \operatorname{Hom}\left(V_{0}, V_{1}\right)$ satisfying equation (6). This equation implies that $v$ is a Lie algebra 2-cocycle representing an element in $H^{2}\left(\mathfrak{g}, \operatorname{Hom}\left(V_{0}, V_{1}\right)\right)$, with the representation $[\mu(\cdot), \cdot]$ of $\mathfrak{g}$ on $\operatorname{Hom}\left(V_{0}, V_{1}\right)$ defined by

$$
\begin{aligned}
{[\mu(\cdot), \cdot](X)(A) } & \triangleq[\mu(X), A] \\
& =\mu_{1}(X) \circ A-A \circ \mu_{0}(X) \text { for all } X \in \mathfrak{g}, A \in \operatorname{Hom}\left(V_{0}, V_{1}\right) .
\end{aligned}
$$

Lemma 4.2. Define $\tilde{v}: \bigwedge^{3}\left(\mathfrak{g} \oplus V_{0}\right) \rightarrow V_{1}$ by

$$
\tilde{v}\left(X_{1}+\xi_{1}, X_{2}+\xi_{2}, X_{3}+\xi_{3}\right)=v\left(X_{1}, X_{2}\right)\left(\xi_{3}\right)+\text { c.p. }
$$

Then $v$ is a 2-cocycle if and only if $\tilde{v}$ is a 3-cocycle where the representation $\tilde{\mu}$ of $\mathfrak{g} \oplus V_{0}$ on $V_{1}$ is given by $\tilde{\mu}(X+\xi)(m)=\mu(X)(m)$.

Proof. By direct computations, for any $X_{i}+\xi_{i} \in \mathfrak{g} \oplus V_{0}$ with $i=1,2,3$, 4, we have

$$
d \tilde{\nu}\left(X_{1}+\xi_{1}, X_{2}+\xi_{2}, X_{3}+\xi_{3}, X_{4}+\xi_{4}\right)=d \nu\left(X_{1}, X_{2}, X_{3}\right)\left(\xi_{4}\right)+\text { c.p. }
$$

The Lie algebra homomorphism $\mu$ from $\mathfrak{g}$ to $\operatorname{End}\left(V_{0}\right) \oplus \operatorname{End}\left(V_{1}\right)$ integrates to a Lie group homomorphism $F_{1}$ from the simply connected Lie group $G$ of $\mathfrak{g}$ to $\mathrm{GL}\left(V_{0}\right) \oplus \mathrm{GL}\left(V_{1}\right)$, with

$$
\mu(X)=\left.\frac{d}{d t}\right|_{t=0} F_{1}(\exp t X) \text { for all } X \in \mathfrak{g} .
$$

Consequently, $\operatorname{Hom}\left(V_{0}, V_{1}\right)$ is a $G$-module with $G$ action

$$
g \cdot A=F_{1}(g) \circ A \circ F_{1}(g)^{-1} \text { for all } g \in G, A \in \operatorname{Hom}\left(V_{0}, V_{1}\right) .
$$

The Lie algebra 2-cocycle $v: \mathfrak{g} \wedge \mathfrak{g} \rightarrow \operatorname{Hom}\left(V_{0}, V_{1}\right)$ can integrate to a smooth Lie group 2-cocycle $\bar{F}_{2}: G \times G \rightarrow \operatorname{Hom}\left(V_{0}, V_{1}\right)$, satisfying

$$
\begin{aligned}
F_{1}\left(g_{1}\right) \circ\left(\bar{F}_{2}\right)\left(g_{2}, g_{3}\right) \circ F_{1}\left(g_{1}\right)^{-1} & -\left(\bar{F}_{2}\right)\left(g_{1} \cdot g_{2}, g_{3}\right)+\left(\bar{F}_{2}\right)\left(g_{1}, g_{2} \cdot g_{3}\right)-\left(\bar{F}_{2}\right)\left(g_{1}, g_{2}\right)=0,
\end{aligned}
$$


and $\bar{F}_{2}\left(1_{G}, 1_{G}\right)=0$. Let us explain how.

The classical theory of cohomology of discrete groups says that the equivalence classes of extensions of $G$ by a $G$ module $M$ are in bijection with the elements of $H^{2}(G, M)$. In our case, the same theory tells us that $H_{\mathrm{sm}}^{2}\left(G, \operatorname{Hom}\left(V_{0}, V_{0}\right)\right)$ classifies the equivalence classes of splitting extensions of $G$ by the $G$-module $\operatorname{Hom}\left(V_{0}, V_{1}\right)$, which is a splitting short exact sequence of Lie groups in which $\operatorname{Hom}\left(V_{0}, V_{1}\right)$ is endowed with an abelian group structure

$$
\operatorname{Hom}\left(V_{0}, V_{1}\right) \rightarrow \hat{G} \rightarrow G .
$$

In a general extension, $\hat{G}$ is a principal bundle over $G$; thus it usually does not permit a smooth lift $G \stackrel{\sigma}{\longrightarrow} \hat{G}$. It permits such a lift if and only if the sequence splits. However in our case, since the abelian group $\operatorname{Hom}\left(V_{0}, V_{1}\right)$ is a vector space, we have $H^{1}\left(X, \operatorname{Hom}\left(V_{0}, V_{1}\right)\right)=0$ for any manifold $X$. The proof makes use of a partition of unity and is similar to the proof showing that $H^{1}(X, \underline{\mathbb{R}})=0$ for the sheaf cohomology. Hence all $\operatorname{Hom}\left(V_{0}, V_{1}\right)$ principal bundles are trivial. Therefore (45) always splits. On the other hand it is well known that when $G$ is simply connected, there is a one-to-one correspondence between extensions of $G$ [Brahic 2010, Theorem 4.15] and extensions of its Lie algebra $\mathfrak{g}$, which in turn are classified by the Lie algebra cohomology $H^{2}\left(\mathfrak{g}, \operatorname{Hom}\left(V_{0}, V_{1}\right)\right)$. Hence in our case the differentiation map $H_{\mathrm{sm}}^{2}\left(G, \operatorname{Hom}\left(V_{0}, V_{1}\right)\right) \rightarrow H^{2}\left(\mathfrak{g}, \operatorname{Hom}\left(V_{0}, V_{1}\right)\right)$ is an isomorphism. Hence $v$ always integrates to a smooth Lie group 2-cocycle unique up to exact 2-cocycles. Then $\bar{F}_{2}\left(1_{G}, 1_{G}\right)=0$ can be arranged too, because we can always modify the section $\sigma: G \rightarrow \hat{G}$ to satisfy $\sigma\left(1_{G}\right)=1_{\hat{G}}$ and the modification of sections results in an exact term. Then combined with (44), it is not hard to see that

$$
\bar{F}_{2}\left(1_{G}, g\right)=\bar{F}_{2}\left(g, 1_{G}\right)=0 \text { for all } g \in G .
$$

Thus $\bar{F}_{2}$ is a normalized 2-cocycle.

Proposition 4.3. For any 2-term representation up to homotopy $(\mu, v)$ of a Lie algebra $\mathfrak{g}$ on the complex $V_{1} \stackrel{0}{\longrightarrow} V_{0}$, there is an associated representation up to homotopy $\left(F_{1}, F_{2}\right)$ of the Lie group $G$ on the complex $V_{1} \stackrel{0}{\longrightarrow} V_{0}$, where $F_{1}$ is the integration of $\mu$ and $F_{2}: G \times G \rightarrow \operatorname{End}\left(V_{0}, V_{1}\right)$ is defined by

$$
F_{2}\left(g_{1}, g_{2}\right)=\bar{F}_{2}\left(g_{1}, g_{2}\right) \circ F_{1}\left(g_{1} \cdot g_{2}\right) .
$$

Proof. Obviously, (31) is satisfied. To see (32) is also satisfied, combine (47) with (44). By the fact that $F_{1}$ is a homomorphism, we obtain

$$
\begin{aligned}
F_{1}\left(g_{1}\right) \circ F_{2}\left(g_{2}, g_{3}\right) \circ F_{1}\left(g_{2} \cdot g_{3}\right)^{-1} \circ F_{1}\left(g_{1}\right)^{-1}-F_{2}\left(g_{1} \cdot g_{2}, g_{3}\right) \circ F_{1}\left(g_{1} \cdot g_{2} \cdot g_{3}\right)^{-1} \\
+F_{2}\left(g_{1}, g_{2} \cdot g_{3}\right) \circ F_{1}\left(g_{1} \cdot g_{2} \cdot g_{3}\right)^{-1}-F_{2}\left(g_{1}, g_{2}\right) \circ F_{1}\left(g_{1} \cdot g_{2}\right)^{-1}=0 .
\end{aligned}
$$


Composing this with $F_{1}\left(g_{1} \cdot g_{2} \cdot g_{3}\right)$ on the right hand side, we obtain (32).

By Proposition 4.3 and Theorem 3.7, we have:

Theorem 4.4. Let $G$ be the simply connected Lie group integrating $\mathfrak{g}$. Then the Lie 2-algebra of string type $\mathbb{R} \stackrel{0}{\longrightarrow} \mathfrak{g} \oplus \mathfrak{g}^{*}$ given by (19) integrates to the Lie 2-group

$$
\begin{gathered}
G \times \mathfrak{g}^{*} \times \mathbb{R} \\
s \| t \\
\qquad \times \mathfrak{g}^{*},
\end{gathered}
$$

in which the source and target are given by

$$
s(g, \xi, m)=t(g, \xi, m)=(g, \xi),
$$

the vertical multiplication $\cdot_{\mathrm{v}}$ is given by

$$
(h, \eta, n) \cdot \mathrm{v}(g, \xi, m)=(g, \xi, m+n), \quad \text { where } h=g, \eta=\xi,
$$

the horizontal multiplication $\cdot_{\mathrm{h}}$ of objects is given by

$$
\left(g_{1}, \xi\right) \cdot \mathrm{h}\left(g_{2}, \eta\right)=\left(g_{1} \cdot g_{2}, \xi+\operatorname{Ad}_{g_{1}}^{*} \eta\right),
$$

the horizontal multiplication $\cdot \mathrm{h}$ of morphisms is given by

$$
\left(g_{1}, \xi, m\right) \cdot{ }_{\mathrm{h}}\left(g_{2}, \eta, n\right)=\left(g_{1} \cdot g_{2}, \xi+\operatorname{Ad}_{g_{1}}^{*} \eta, m+n\right),
$$

the inverse map inv is given by

$$
\operatorname{inv}(g, \xi)=\left(g^{-1},-\operatorname{Ad}_{g^{-1}}^{*} \xi\right), \quad \operatorname{inv}(g, \xi, m)=\left(g^{-1},-\operatorname{Ad}_{g^{-1}}^{*} \xi,-m\right),
$$

the identity object is $\left(1_{G}, 0\right)$, and the associator

$$
a_{\left(g_{1}, \xi\right),\left(g_{2}, \eta\right),\left(g_{3}, \gamma\right)}:\left(\left(g_{1}, \xi\right) \cdot \mathrm{h}\left(g_{2}, \eta\right)\right) \cdot{ }_{\mathrm{h}}\left(g_{3}, \gamma\right) \rightarrow\left(g_{1}, \xi\right) \cdot{ }_{\mathrm{h}}\left(\left(g_{2}, \eta\right) \cdot{ }_{\mathrm{h}}\left(g_{3}, \gamma\right)\right)
$$

is given by

$$
a_{\left(g_{1}, \xi\right),\left(g_{2}, \eta\right),\left(g_{3}, \gamma\right)}=\left(g_{1} \cdot g_{2} \cdot g_{3}, \xi+\operatorname{Ad}_{g_{1}}^{*} \eta+\operatorname{Ad}_{g_{1} \cdot g_{2}}^{*} \gamma, F_{2}\left(g_{1}, g_{2}\right)(\gamma)\right) .
$$

All the other structures are identity isomorphisms.

Proof. Since $F_{1}$ is a usual associative action, we may modify the unit (40) given in Theorem 3.7 to be the identity natural transformation. It turns out that (48) is a special Lie 2-group and is represented by $\left(G \ltimes \mathfrak{g}^{*}, \mathbb{R}\right.$, Id, $\left.\widetilde{F}_{2}\right)$, where $G \ltimes \mathfrak{g}^{*}$ is the semidirect product with the coadjoint action of $G$ on $\mathfrak{g}^{*}$, Id is the constant map $G \ltimes \mathfrak{g}^{*} \rightarrow \operatorname{Aut}(\mathbb{R})$ that maps everything to $\operatorname{Id} \in \operatorname{Aut}(\mathbb{R})$, and $\widetilde{F}_{2}$ is given by

(50) $\widetilde{F}_{2}\left(\left(g_{1}, \xi_{1}\right),\left(g_{2}, \xi_{2}\right),\left(g_{3}, \xi_{3}\right)\right)=F_{2}\left(g_{1}, g_{2}\right)\left(\xi_{3}\right)=\bar{F}_{2}\left(g_{1}, g_{2}\right) \circ F_{1}\left(g_{1} \cdot g_{2}\right)\left(\xi_{3}\right)$. 
Since $\bar{F}_{2}$ is normalized, so is $\widetilde{F}_{2}$. The Lie 2 -algebra of string type $\mathbb{R} \stackrel{0}{\rightarrow} \mathfrak{g} \oplus \mathfrak{g}^{*}$ is skeletal and is represented by $\left(\mathfrak{g} \oplus \mathfrak{g}^{*}, \mathbb{R}, 0, \tilde{v}\right)$, where $\tilde{v}$ is given by (20). Thus to show that our Lie 2-group is one of its integrations, we only need to show that the differential of the Lie group 3-cocycle $\widetilde{F}_{2}$ is the Lie algebra 3-cocycle $\tilde{v}$. By direct computations [Brylinski 1993, Lemma 7.3.9], we have

$$
\begin{aligned}
& \left.\frac{\partial^{3}}{\partial_{t_{1}} \partial_{t_{2}} \partial_{t_{3}}}\right|_{t_{t_{i}}=0} \epsilon(\sigma) \widetilde{F}_{2}\left(\left(e^{t_{\sigma(1)} X_{\sigma(1)}}, t_{\sigma(1)} \xi_{\sigma(1)}\right),\left(e^{t_{\sigma(2)} X_{\sigma(2)}}, t_{\sigma(2)} \xi_{\sigma(2)}\right),\left(e^{t_{\sigma(3)} X_{\sigma(3)}}, t_{\sigma(3)} \xi_{\sigma(3)}\right)\right) \\
& =\left.\frac{\partial^{3}}{\partial_{t_{1}} \partial_{t_{2}} \partial_{t_{3}}}\right|_{t_{i}=0}\left(\bar{F}_{2}\left(e^{t_{1} X_{1}}, e^{t_{2} X_{2}}\right) \circ F_{1}\left(e^{t_{1} X_{1}} \cdot e^{t_{2} X_{2}}\right)\left(t_{3} \xi_{3}\right)\right)+\text { c.p. } \\
& =\left.\frac{\partial^{2}}{\partial_{t_{1}} \partial_{t_{2}}}\right|_{t_{i}=0}\left(\bar{F}_{2}\left(e^{t_{1} X_{1}}, e^{t_{2} X_{2}}\right) \circ F_{1}\left(e^{t_{1} X_{1}} \cdot e^{t_{2} X_{2}}\right)\left(\xi_{3}\right)\right)+\text { c.p. } \\
& =\left.\frac{\partial}{\partial_{t_{1}}}\right|_{t_{1}=0}\left(\left.\frac{\partial}{\partial_{t_{2}}}\right|_{t_{2}=0} \bar{F}_{2}\left(e^{t_{1} X_{1}}, e^{t_{2} X_{2}}\right) \circ F_{1}\left(e^{t_{1} X_{1}}\right)\left(\xi_{3}\right)\right. \\
& \left.\qquad \quad+\left.\bar{F}_{2}\left(e^{t_{1} X_{1}}, 1_{G}\right) \circ \frac{\partial}{\partial_{t_{2}}}\right|_{t_{2}=0} F_{1}\left(e^{t_{1} X_{1}} \cdot e^{t_{2} X_{2}}\right)\right)+ \text { c.p. } \\
& =\left.\frac{\partial}{\partial_{t_{1}}} \frac{\partial}{\partial_{t_{2}}}\right|_{t_{i}=0} \bar{F}_{2}\left(e^{t_{1} X_{1}}, e^{t_{2} X_{2}}\right)\left(\xi_{3}\right)+\left.\left.\frac{\partial}{\partial_{t_{2}}}\right|_{t_{2}=0} \bar{F}_{2}\left(1_{G}, e^{t_{2} X_{2}}\right) \circ \frac{\partial}{\partial_{t_{1}}}\right|_{t_{1}=0} F_{1}\left(e^{t_{1} X_{1}}\right)\left(\xi_{3}\right) \\
& =v\left(X_{1}, X_{2}\right)\left(\xi_{3}\right)+\text { c.p.p. } \quad \text { (by (46)) } \\
& =\tilde{v}\left(X_{1}+\xi_{1}, X_{2}+\xi_{2}, X_{3}+\xi_{3}\right) \quad(\text { by }(20)),
\end{aligned}
$$

which completes the proof.

Corollary 4.5. If Lie algebra $\mathfrak{g}$ is semisimple, the Lie group 3-cocycle $\widetilde{F}_{2}$ is not exact, that is, $\left[\widetilde{F}_{2}\right] \neq 0$ in $H_{\text {sm }}^{3}\left(G \ltimes \mathfrak{g}^{*}, \mathbb{R}\right)$.

Proof. By Theorem 4.4, the differentiation of the Lie group 3-cocycle $\widetilde{F}_{2}$ is the Lie algebra 3-cocycle $\tilde{v}$. We only need to show that when $\mathfrak{g}$ is semisimple, the Lie algebra 3-cocycle $\tilde{v}$ is not exact. This fact is proved in Proposition 2.13.

Remark 4.6. Since $G \ltimes \mathfrak{g}^{*}$ is a fibration over $G$, the spectral sequence with $E_{2}^{p, q}=$ $H_{\mathrm{sm}}^{p}\left(G, H_{\mathrm{sm}}^{q}\left(\mathfrak{g}^{*}, \mathbb{R}\right)\right)$ calculates the group cohomology $H_{\mathrm{sm}}^{3}\left(G \ltimes \mathfrak{g}^{*}, \mathbb{R}\right)$. Since $\mathfrak{g}^{*}$ is an abelian group, we have $H_{\mathrm{sm}}^{q}\left(\mathfrak{g}^{*}, \mathbb{R}\right)=\bigwedge^{q} \mathfrak{g}^{*}$. Thus when $G$ is compact, $H_{\mathrm{sm}}^{p}\left(G, H_{\mathrm{sm}}^{q}\left(\mathfrak{g}^{*}, \mathbb{R}\right)\right)=\left(\bigwedge^{q} \mathfrak{g}^{*}\right)^{G}$ if $p=0$ and 0 otherwise, where $\left(\bigwedge^{q} \mathfrak{g}^{*}\right)^{G}$ denotes the set of invariant elements of $\wedge^{q} \mathfrak{g}^{*}$ under the coadjoint action of $G$. Thus when $G$ is compact, $H_{\mathrm{sm}}^{3}\left(G \ltimes \mathfrak{g}^{*}, \mathbb{R}\right)=\left(\bigwedge^{3} g^{*}\right)^{G} \neq 0$ because the Cartan 3-form is an element of $\left(\bigwedge^{3} g^{*}\right)^{G}$.

Our 2-cocycle $\bar{F}_{2}$ is unique only up to exact terms. Hence by Theorem 3.3, we need this lemma to verify that our construction is unique up to isomorphism: 
Lemma 4.7. If $\bar{F}_{2}=\mathrm{d} \alpha$ is exact, then $\widetilde{F}_{2}=\mathrm{d} \beta$ is also exact with

$$
\beta\left(\left(g_{1}, \xi_{1}\right),\left(g_{2}, \xi_{2}\right)\right)=\alpha\left(g_{1}\right) F_{1}\left(g_{1}\right)\left(\xi_{2}\right) .
$$

Proof. It is a direct calculation. Since $\bar{F}_{2}=\mathrm{d} \alpha$, we have

$$
\bar{F}_{2}\left(g_{1}, g_{2}\right)=F_{1}\left(g_{1}\right) \alpha\left(g_{2}\right) F_{1}\left(g_{1}\right)^{-1}-\alpha\left(g_{1} g_{2}\right)+\alpha\left(g_{1}\right) .
$$

From the definition of $F_{2}$, we know that

$$
F_{2}\left(g_{1}, g_{2}\right)=F_{1}\left(g_{1}\right) \alpha\left(g_{2}\right) F_{1}\left(g_{1}\right)^{-1} F_{1}\left(g_{1} g_{2}\right)-\alpha\left(g_{1} g_{2}\right) F_{1}\left(g_{1} g_{2}\right)+\alpha\left(g_{1}\right) F_{1}\left(g_{1} g_{2}\right) \text {. }
$$

By (50), we have

$$
\begin{aligned}
& \widetilde{F}_{2}\left(\left(g_{1}, \xi_{1}\right),\left(g_{2}, \xi_{2}\right),\left(g_{3}, \xi_{3}\right)\right) \\
& =F_{1}\left(g_{1}\right) \alpha\left(g_{2}\right) F_{1}\left(g_{1}\right)^{-1} F_{1}\left(g_{1} g_{2}\right)\left(\xi_{3}\right)-\alpha\left(g_{1} g_{2}\right) F_{1}\left(g_{1} g_{2}\right)\left(\xi_{3}\right)+\alpha\left(g_{1}\right) F_{1}\left(g_{1} g_{2}\right)\left(\xi_{3}\right) \\
& =\mathrm{d} \beta\left(\left(g_{1}, \xi_{1}\right),\left(g_{2}, \xi_{2}\right),\left(g_{3}, \xi_{3}\right)\right),
\end{aligned}
$$

since $F_{1}$ is a group homomorphism.

Remark 4.8. Our Lie 2-group as a stacky group has the underlying differential stack $G \times \mathfrak{g}^{*} \times B \mathbb{R}$. Thus it is $0,1,2$-connected (that is, it has $\pi_{0}=\pi_{1}=\pi_{2}=0$ ) since $\pi_{2}(B \mathbb{R})=\pi_{1}(\mathbb{R})=0$ and $\pi_{1}(B \mathbb{R})=\pi_{0}(\mathbb{R})=0$. Thus it is the unique $0,1,2$ connected stacky Lie group integrating the Lie 2-algebra of string type $\mathbb{R} \stackrel{0}{\longrightarrow} \mathfrak{g} \oplus \mathfrak{g}^{*}$ in the sense of [Zhu 2007].

\section{Acknowledgements}

We give our warmest thanks to Zhang-Ju Liu, Jiang-Hua Lu, Giorgio Trentinagia and Marco Zambon for useful comments and discussion. Y. Sheng thanks the Courant Research Centre "Higher Order Structures" at Göttingen University, where this work was during his visit there.

\section{References}

[Abad 2008] C. Abad, Representation up to homotopy and cohomology of classifying spaces, thesis, Utrecht University, 2008.

[Abad and Crainic 2009] C. Abad and M. Crainic, "Representations up to homotopy of Lie algebroids", preprint, 2009. arXiv 0901.0319

[Baez and Crans 2004] J. C. Baez and A. S. Crans, "Higher-dimensional algebra, VI: Lie 2-algebras", Theory Appl. Categ. 12 (2004), 492-538. MR 2005m:17039 Zbl 1057.17011

[Baez and Lauda 2004] J. C. Baez and A. D. Lauda, "Higher-dimensional algebra, V: 2-groups", Theory Appl. Categ. 12 (2004), 423-491. MR 2005m:18005 Zbl 1056.18002

[Baez and Rogers 2010] J. C. Baez and C. L. Rogers, "Categorified symplectic geometry and the string Lie 2-algebra”, Homology, Homotopy Appl. 12 (2010), 221-236. MR 2638872 Zbl 05700809

[Blohmann 2008] C. Blohmann, "Stacky Lie groups", Int. Math. Res. Not. 2008 (2008), Art. ID rnn 082. MR 2010b:58023 Zbl 1154.53053 
[Brahic 2010] O. Brahic, "Extensions of Lie brackets", J. Geom. Phys. 60:2 (2010), 352-374. MR 2587399 Zbl 05675303

[Brylinski 1993] J.-L. Brylinski, Loop spaces, characteristic classes and geometric quantization, Progress in Mathematics 107, Birkhäuser, Boston, MA, 1993. MR 94b:57030 Zbl 0823.55002

[Chen and Liu 2010] Z. Chen and Z.-J. Liu, "Omni-Lie algebroids”, J. Geom. Phys. 60:5 (2010), 799-808. MR 2608529 Zbl 05702811

[Chen et al. 2008] Z. Chen, Z.-J. Liu, and Y.-H. Sheng, "Dirac structures of omni-Lie algebroids", preprint, 2008. arXiv 0802.3819

[Chen et al. 2010] Z. Chen, Z.-J. Liu, and Y.-H. Sheng, "E-Courant algebroids", Int. Math. Res. Not. 22 (2010), 4334-4376.

[Getzler 2009] E. Getzler, "Lie theory for nilpotent $L_{\infty}$-algebras", Ann. of Math. (2) 170:1 (2009), 271-301. MR 2010g:17026 Zbl 05578961

[Henriques 2008] A. Henriques, "Integrating $L_{\infty}$-algebras", Compos. Math. 144:4 (2008), 10171045. MR 2441255 Zbl 1152.17010

[Liu et al. 1997] Z.-J. Liu, A. Weinstein, and P. Xu, "Manin triples for Lie bialgebroids", J. Differential Geom. 45:3 (1997), 547-574. MR 98f:58203 Zbl 0885.58030

[Lu and Weinstein 1990] J.-H. Lu and A. Weinstein, "Poisson Lie groups, dressing transformations, and Bruhat decompositions”, J. Differential Geom. 31:2 (1990), 501-526. MR 91c:22012 Zbl 0673.58018

[Roytenberg 2002] D. Roytenberg, "On the structure of graded symplectic supermanifolds and Courant algebroids", pp. 169-185 in Quantization, Poisson brackets and beyond (Manchester, 2001), edited by T. Voronov, Contemp. Math. 315, Amer. Math. Soc., Providence, RI, 2002. MR 2004i: 53116 Zbl 1036.53057

[Roytenberg and Weinstein 1998] D. Roytenberg and A. Weinstein, "Courant algebroids and strongly homotopy Lie algebras”, Lett. Math. Phys. 46:1 (1998), 81-93. MR 2000c:17036 Zbl 0946.17006

[Schommer-Pries 2010] C. Schommer-Pries, "A finite dimensional string 2-group", preprint, 2010. arXiv 0911.2483

[Schommer-Pries et al. $\geq 2011]$ C. Schommer-Pries, C. Wockel, and C. Zhu, "Integration of central extensions of Lie 2-algebras", in preparation.

[Ševera 2005] P. Ševera, "Some title containing the words "homotopy" and "symplectic", e.g. this one", pp. 121-137 in Travaux mathématiques, XVI (Luxemboug, 2004), edited by C. M.-B. dn Norbert Poncin and M. Schlichenmaier, Trav. Math. 16, Univ. Luxemb., 2005. MR 2007f:53107 Zbl 1102.58010

[Sheng and Zhu 2010] Y. Sheng and C. Zhu, "Integration of semidirect product Lie 2-algebras", preprint, 2010. arXiv 1003.1348

[Stasheff 1992] J. Stasheff, "Differential graded Lie algebras, quasi-Hopf algebras and higher homotopy algebras", pp. 120-137 in Quantum groups (Leningrad, 1990), edited by P. P. Kulish, Lecture Notes in Math. 1510, Springer, Berlin, 1992. MR 93j:17055 Zbl 0758.17010

[Zhu 2007] C. Zhu, "Lie II theorem for Lie algebroids via stacky Lie groupoids", preprint, 2007. arXiv math/0701024

[Zhu 2009] C. Zhu, "n-groupoids and stacky groupoids", Int. Math. Res. Not. 2009:21 (2009), 40874141. MR 2549951 Zbl 1180.22006

Received October 14, 2009. Revised November 25, 2009. 
YUNHE SHENG

DEPARTMENT OF MATHEMATICS

JILIN UNIVERSITY

ChangChun, JiLin 130012

CHINA

ysheng888@gmail.com

Chenchang Zhu

Courant Research Centre "Higher Order Structures"

MATHEMATISCHES INSTITUT

UNIVERSITY OF GÖTTINGEN

BUNSENSTRASSE 3-5

D-37073 GÖTTINGEN

GERMANY

zhu@uni-math.gwdg.de 


\title{
PACIFIC JOURNAL OF MATHEMATICS
}

\author{
http://www.pjmath.org \\ Founded in 1951 by \\ E. F. Beckenbach (1906-1982) and F. Wolf (1904-1989)
}

\section{EDITORS}

V. S. Varadarajan (Managing Editor)

Department of Mathematics

University of California

Los Angeles, CA 90095-1555

pacific@math.ucla.edu

Vyjayanthi Chari

Department of Mathematics

University of California

Riverside, CA 92521-0135

chari@math.ucr.edu

Robert Finn

Department of Mathematics Stanford University

Stanford, CA 94305-2125

finn@math.stanford.edu

Kefeng Liu

Department of Mathematics

University of California

Los Angeles, CA 90095-1555

liu@math.ucla.edu
Darren Long

Department of Mathematics

University of California

Santa Barbara, CA 93106-3080

long@math.ucsb.edu

Jiang-Hua Lu

Department of Mathematics

The University of Hong Kong

Pokfulam Rd., Hong Kong jhlu@maths.hku.hk

Alexander Merkurjev

Department of Mathematics

University of California

Los Angeles, CA 90095-1555

merkurev@math.ucla.edu
Sorin Popa

Department of Mathematics University of California

Los Angeles, CA 90095-1555 popa@math.ucla.edu

Jie Qing

Department of Mathematics

University of California

Santa Cruz, CA 95064

qing@cats.ucsc.edu

Jonathan Rogawski

Department of Mathematics

University of California

Los Angeles, CA 90095-1555

jonr@math.ucla.edu

\section{PRODUCTION}

pacific@math.berkeley.edu

\begin{abstract}
Silvio Levy, Scientific Editor Matthew Cargo, Senior Production Editor
\end{abstract}
ACADEMIA SINICA, TAIPEI

CALIFORNIA INST. OF TECHNOLOGY

INST. DE MATEMÁTICA PURA E APLICADA

KEIO UNIVERSITY

MATH. SCIENCES RESEARCH INSTITUTE

NEW MEXICO STATE UNIV.

OREGON STATE UNIV.

\section{SUPPORTING INSTITUTIONS}

STANFORD UNIVERSITY
UNIV. OF BRITISH COLUMBIA
UNIV. OF CALIFORNIA, BERKELEY
UNIV. OF CALIFORNIA, DAVIS
UNIV. OF CALIFORNIA, LOS ANGELES
UNIV. OF CALIFORNIA, RIVERSIDE
UNIV. OF CALIFORNIA, SAN DIEGO
UNIV. OF CALIF., SANTA BARBARA

UNIV. OF CALIF., SANTA CRUZ

UNIV. OF MONTANA

UNIV. OF OREGON

UNIV. OF SOUTHERN CALIFORNIA

UNIV. OF UTAH

UNIV. OF WASHINGTON

WASHINGTON STATE UNIVERSITY

These supporting institutions contribute to the cost of publication of this Journal, but they are not owners or publishers and have no responsibility for its contents or policies.

See inside back cover or www.pjmath.org for submission instructions.

The subscription price for 2011 is US \$420/year for the electronic version, and \$485/year for print and electronic.

Subscriptions, requests for back issues from the last three years and changes of subscribers address should be sent to Pacific Journal of Mathematics, P.O. Box 4163, Berkeley, CA 94704-0163, U.S.A. Prior back issues are obtainable from Periodicals Service Company, 11 Main Street, Germantown, NY 12526-5635. The Pacific Journal of Mathematics is indexed by Mathematical Reviews, Zentralblatt MATH, PASCAL CNRS Index, Referativnyi Zhurnal, Current Mathematical Publications and the Science Citation Index.

The Pacific Journal of Mathematics (ISSN 0030-8730) at the University of California, c/o Department of Mathematics, 969 Evans Hall, Berkeley, CA 94720-3840, is published monthly except July and August. Periodical rate postage paid at Berkeley, CA 94704, and additional mailing offices. POSTMASTER: send address changes to Pacific Journal of Mathematics, P.O. Box 4163, Berkeley, CA 94704-0163.

PJM peer review and production are managed by EditFLOW ${ }^{\mathrm{TM}}$ from Mathematical Sciences Publishers.

PUBLISHED BY PACIFIC JOURNAL OF MATHEMATICS

at the University of California, Berkeley 94720-3840

A NON-PROFIT CORPORATION

Typeset in LATEX

Copyright $(02011$ by Pacific Journal of Mathematics 


\section{PACIFIC JOURNAL OF MATHEMATICS}

Volume $249 \quad$ No. $1 \quad$ January 2011

Metabelian SL $(n, \mathbb{C})$ representations of knot groups, II: Fixed points

HANS U. BODEN and STEFAN FRIEDL

Lewis-Zagier correspondence for higher-order forms

ANTON DEITMAR

Topology of positively curved 8-dimensional manifolds with symmetry

ANAND DESSAI

Strong Kähler with torsion structures from almost contact manifolds

MARISA FERnÁNDEZ, ANNA FINO, LUIS UGARTE and RAQUEL

VILLACAMPA

Connections between Floer-type invariants and Morse-type invariants of Legendrian knots

MICHAEL B. HENRY

A functional calculus for unbounded generalized scalar operators on Banach spaces

DRAGOLJUB KEČKIĆ and ĐORĐE KRTINIĆ

Geometric formality of homogeneous spaces and of biquotients

D. KoTSCHICK and S. TERZIĆ

Positive solutions for a nonlinear third order multipoint boundary value problem

Yang LiU, Zhang Weiguo, Liu Xiping, Shen Chunfang and Chen HUA

The braid group surjects onto $G_{2}$ tensor space

SCOTT MORRISON

Analogues of the Wiener Tauberian and Schwartz theorems for radial functions on symmetric spaces

E. K. NARAyAnAn and Alladi Sitaram

Semidirect products of representations up to homotopy

Yunhe SHENG and Chenchang ZHU

Homology sequence and excision theorem for Euler class group

YONG YANG 